\title{
The VELVET Complex in the Gray Mold Fungus Botrytis cinerea: Impact of BcLAE1 on Differentiation, Secondary Metabolism, and Virulence
}

\author{
Julia Schumacher, ${ }^{1}$ Adeline Simon, ${ }^{2}$ Kim C. Cohrs, ${ }^{1}$ Stefanie Traeger, ${ }^{1}$ Antoine Porquier, ${ }^{2,3}$ \\ Bérengère Dalmais, ${ }^{2}$ Muriel Viaud, ${ }^{2}$ and Bettina Tudzynski ${ }^{1}$ \\ ${ }^{1}$ IBBP, WWU Münster, Schlossplatz 8, 48143 Münster, Germany; ${ }^{2}$ BIOGER, INRA, Avenue Lucien Brétignières, 78850 \\ Grignon, France; ${ }^{3}$ Université Paris-Sud, 91405 Orsay, France
}

Submitted 24 December 2014. Accepted 21 January 2015.

\begin{abstract}
Botrytis cinerea, the gray mold fungus, is an important plant pathogen. Field populations are characterized by variability with regard to morphology, the mode of reproduction (conidiation or sclerotia formation), the spectrum of secondary metabolites (SM), and virulence. Natural variation in bcvell encoding the ortholog of Aspergillus nidulans VeA, a member of the VELVET complex, was previously shown to affect lightdependent differentiation, the formation of oxalic acid (OA), and virulence. To gain broader insight into the $B$. cinerea VELVET complex, an ortholog of A. nidulans LaeA, BcLAE1, a putative interaction partner of BcVEL1, was studied. BcVEL1 but not its truncated versions interacts with BcLAE1 and BcVEL2 (VelB ortholog). In accordance with the expected common as well as specific functions of BcVEL1 and BcLAE1, the deletions of both genes result in similar though not identical phenotypes. Both mutants lost the ability to produce $\mathrm{OA}$, to colonize the host tissue, and to form sclerotia. However, mutants differ with regard to aerial hyphae and conidia formation. Genome-wide expression analyses revealed that BCVEL1 and BcLAE1 have common and distinct target genes. Some of the genes that are underexpressed in both mutants, e.g., those encoding SM-related enzymes, proteases, and carbohydrate-active enzymes, may account for their reduced virulence.
\end{abstract}

Secondary metabolites (SM) are small molecules that, unlike primary metabolites, are not directly required for growth of the producing organism. Fungal SM biosynthetic genes are typically located adjacent to each other and exhibit similar patterns of expression (coregulation). These clusters usually include a gene encoding the key enzyme (KE) responsible for synthesis of the raw product and genes encoding enzymes for further modifications and for the export of the compound. The regulation of SM gene clusters occurs at several levels, i.e., by pathway-specific transcription factors (TF) that are usually encoded by a cluster gene, by broad domain TF that integrate

Current address for S. Traeger: RUB, Universitätsstr. 150, 44801 Bochum, Germany

Corresponding authors: J. Schumacher; E-mail: julia.schumacher@wwu.de; M. Viaud; E-mail: viaud@ versailles.inra.fr

*The $\boldsymbol{e}$-Xtra logo stands for "electronic extra" and indicates that two supplementary figures and two supplementary tables are published online.

๑) 2015 The American Phytopathological Society environmental signals, and by global regulators that affect gene expression via chromatin remodeling (Brakhage 2013; Hoffmeister and Keller 2007; Wiemann and Keller 2014).

LaeA (loss of aflR expression), a putative methyltransferase, was first identified in Aspergillus nidulans as a regulator of SM gene clusters (Bok and Keller 2004; Bok et al. 2005). Later, LaeA was shown to form a protein (VELVET) complex with the fungal regulatory proteins $\mathrm{VeA}$ and VelB that coordinates development and secondary metabolism in response to light (Bayram et al. 2008). In the meantime, VeA and LaeA orthologs in other species, including Penicillium chrysogenum (Hoff et al. 2010), Fusarium fujikuroi (Wiemann et al. 2010), and Cochliobolus heterostrophus (Wu et al. 2012), have been characterized, demonstrating that their general functions are conserved among ascomycetes (Bayram and Braus 2012).

Botrytis cinerea is the causal agent of gray mold diseases in numerous dicotyledonous plant species including high-value crops such as grapevine, strawberry, raspberry, and ornamental flowers. Wide ranges of symptoms are provoked by $B$. cinerea, from brown spots to full-scale soft rotting, the latter accompanied by the appearance of gray masses of conidia (van Kan 2006; Williamson et al. 2007). SM may be connected to the life cycle of $B$. cinerea in different ways. They may contribute to virulence by killing plant cells, allowing for subsequent colonization of the dead tissue by the fungus, they may help to compete with other organisms, and they may protect the reproductive structures, i.e., conidia, sclerotia, and apothecia from abiotic and biotic stresses such as UV light and mycoparasitism.

The genome of $B$. cinerea comprises 44 genes encoding SM KE including 21 polyketide synthases (PKS), nine nonribosomal peptide synthetases (NRPS), six sesquiterpene cyclases, one chalcone synthase, two dimethylallyl tryptophan synthases (DMATS), and five diterpene cyclases (Amselem et al. 2011). To date, only a small number of SM have been identified and linked to specific gene clusters in the genome of B. cinerea. i) Botrydial (BOT) is a sesquiterpenoid (Collado et al. 2007) that triggers plant cell death via induction of the hypersensitive response (Rossi et al. 2011). Its biosynthesis relies on a cluster of five coregulated genes, including the KEencoding gene bcbot $2 /$ bcstcl (Pinedo et al. 2008; Siewers et al. 2005). ii) Botcinic acid (BOA) and its derivatives (botcinins) are combined phytotoxic polyketides (Tani et al. 2005, 2006) whose biosynthesis depends on two PKS, BcBOA6 and BcBOA9, which belong to two clusters of coregulated genes (Dalmais et al. 2011; Massaroli et al. 2013). iii) Abscisic acid (ABA) is a plant hormone and belongs to the group of 
sesquiterpenoids (Oritani and Kiyota 2003). While, in higher plants, ABA biosynthesis is mediated via the carotenoid pathway, $B$. cinerea synthesizes ABA directly from farnesyl diphosphate via different oxidative steps. A cluster of four involved genes was described (Siewers et al. 2004, 2006). iv) Oxalic acid (OA) is a compound that is produced via the oxaloacetate acetylhydrolase in B. cinerea and Sclerotinia sclerotiorum. Loss of OA formation results in avirulent mutants in $S$. sclerotiorum (Godoy et al. 1990; Liang et al. 2014), but bcoahA mutants are still able to colonize the host tissue (Han et al. 2007; Stefanato et al. 2008). v) Bikaverin (BIK) is a red polyketide pigment found in some Fusarium species, e.g., F. fujikuroi, in which the BIK biosynthesis and the involved genes have been intensively investigated (Wiemann et al. 2009). Recent studies revealed that an ancestor of $B$. cinerea acquired the gene cluster via horizontal gene transfer from Fusarium spp. (Campbell et al. 2012; Schumacher et al. 2013). vi) 1,8-Dihydroxynaphthalene (DHN)-melanin is a polyketide derivative accumulating in the conidiophores, conidia, and sclerotia of $B$. cinerea, giving them their characteristic dark color (Doss et al. 2003; Zeun and Buchenauer 1985). In contrast to other DHN-melanin forming fungi, $B$. cinerea possesses two highly similar KE-encoding genes (bcpks12, bcpks13), while single copies are present for the other melanogenic genes (Kroken et al. 2003; Schumacher et al. 2014). vii) Carotenoids (CAR) are tetraterpenoids that are produced by plants and fungi to protect cells from free radicals and singlet oxygen (Gao and Garcia-Pichel 2011). A cluster of four light-induced genes was recently identified in $B$. cinerea that shares similarity with the studied CAR gene cluster in F. fujikuroi (Schumacher et al. 2014).

Importantly, the property to produce the mentioned SM varies among $B$. cinerea wild-type strains. ABA and BIK are only formed by a few strains (Schumacher et al. 2013; Siewers et al. 2004), and not all wild-type strains produce OA, BOT, and BOA (Reino et al. 2004; Schumacher et al. 2012). As with the production of SM, the ability to differentiate the reproductive structures (conidia, sclerotia) also varies among $B$. cinerea wild-type strains. In the majority of strains, the mode of (asexual) reproduction is determined by light conditions; conidia are formed in the light, sclerotia in the dark (Williamson et al. 2007). However, in a number of strains, the lightdependent regulation of differentiation is abolished; they produce either conidia, sclerotia, or sterile hyphae in any light condition (Canessa et al. 2013; Paul 1929; Stewart and Long 1987). Recently, single nucleotide polymorphisms (SNP) resulting in truncated BcVEL1 (VeA) proteins of 184 amino acids (aa) and 100 aa, compared with the 575-aa protein found in the light-responsive wild-type strain B05.10, were identified by map-based cloning approaches as the genetic basis for persistent conidiation, loss of sclerotia formation, reduced virulence, and loss of OA formation of wild-type strains T4 and 1750 (Schumacher et al. 2012, 2013). The characterization of bcvell deletion mutants in the B05.10 background, furthermore, revealed the impact of BcVEL1 on DHN-melanin formation, the response to oxidative stress, nitrate assimilation, and the regulation of genes encoding proteases, transporters, and glycoside hydrolases (Schumacher et al. 2012). Similar phenotypes regarding conidiation, melanin biosynthesis, hypersensitivity to oxidative stress, and virulence have been reported for bcvell and bcvel2 (VelB ortholog) mutants in the strain 38B1 (Yang et al. 2013). However, this study is based on incorrect gene models of both VELVET proteins (VeA,294 instead of 575 aa; VelB, 345 instead of 451 aa).

In this work, we studied the functions of BcLAE1, another putative member of the VELVET complex, in regulation of differentiation, virulence, and SM formation. We demonstrate that full-length BcVEL1, indeed, interacts with
BcLAE1 and BcVEL2, forming a VELVET-like complex. Our data demonstrate that BcLAE1 is dispensable for growth under standard conditions but is required for OA formation, for tolerance of oxidative stress, and for full virulence on French bean, as shown previously for BcVEL1. Both gene deletions decoupled the differentiation programs from light control; however, the deletion of bclael caused a milder phenotype. Genome-wide expression analyses revealed sets of common and unique target genes of BcVEL1 and BcLAE1 that are enriched for genes encoding SM-related enzymes, (secreted) carbohydrate-active enzymes, and proteases. In sum, this study demonstrates that both proteins are global regulators of differentiation and secondary metabolism in the gray mold fungus and highlights the role of the $B$. cinerea VELVET complex in virulence, possibly due to the regulation of a wide range of enzymes involved in SM and plant cell degradation.

\section{RESULTS}

\section{Identification of the LaeA ortholog in B. cinerea.}

Several candidates were identified in the B. cinerea B05.10 genome database (Broad Institute) by BlastP analyses using the sequence of $A$. nidulans LaeA (GenBank accession number AAQ95166; Bok and Keller 2004) as query. Best hits were the annotated proteins BC1G_15168 and BC1G_15169 from the first sequencing approach by Syngenta (Amselem et al. 2011) and B0510_1382 from the resequencing approach by Staats and van Kan (2012), which cover the same genomic locus. As both automatic gene predictions were incorrect (Supplementary Fig. S1), the annotation was revised by cDNA sequencing (GenBank accession number KP099423). Based on this sequence, the open reading frame (ORF) of bclae 1 comprises $1,572 \mathrm{bp}$ and is interrupted by six introns of $181,65,73,57,64$, and $148 \mathrm{bp}$ and encodes a protein of 327 aa. Phylogenetic analysis indicated that BcLAE1 more closely relates with the ortholog of the dothideomycete C. heterostrophus (54\% identity, $65 \%$ positives) than with orthologs from the eurotiomycetes, e.g., A. nidulans $(42 \%$ identity, $61 \%$ positives), and the sordariomycetes, e.g., Trichoderma reesei (40\% identity, 59\% positives) (National Center for Biotechnology Information [NCBI] BlastP Align). The methyltransferase domain including the conserved binding sites for $S$-adenosylmethionine comprises the amino acids 85 to 177 of BcLAE1.

\section{BcLAE1, BcVEL1, and BcVEL2 form a complex in some but not all $B$. cinerea strains.}

In A. nidulans, LaeA forms a stable complex with the VELVET proteins VeA and VelB in the nucleus, whereby VeA functions as a bridging factor by interacting with VelB (via the $\mathrm{N}$-terminal region) and LaeA (via the C-terminal region) (Bayram et al. 2008). To ascertain analogous protein-protein interactions of the $B$. cinerea orthologs (BcLAE1 [327 aa], BcVEL1 [575 aa], and BcVEL2 [451 aa]), a direct yeast twohybrid approach was performed. The full-length proteins were fused to the GAL4 binding and activation domains, and yeast cells expressing the different protein combinations were inspected for HIS3 and $L a c Z$ reporter gene activities. The assays indicated that BcVEL1 interacts with BcLAE1 as well as with BcVEL2 (Fig. 1A). To consider the natural mutations of bcvell in B. cinerea wild-type strains generating at least two further alleles, bcvell ${ }^{\mathrm{T} 4}$ and bcvel $^{1750}$, the respective proteins of 184 and 100 aa were included in the yeast two-hybrid assay. The truncated proteins failed to interact with both BcLAE1 and BcVEL2, suggesting that no trimeric complex is formed in wild-type strains expressing these alleles. The fact that the 
corresponding C-termini of BcVEL1 (186 to 575 aa, 102 to 575 aa) still interact with BcLAE1 but not with BcVEL2 could imply that the N-terminal VELVET domain (23 to 220 aa) that is disrupted in all truncated BcVEL1 versions is essential for interaction with BcVEL2 but dispensable for interaction with BcLAE1. In sum, our data suggest that a heterotrimeric VELVET complex exists in $B$. cinerea strains that express a full-length BcVEL1, such as the commonly used wild-type strain B05.10.

\section{Subcellular localizations of BcVEL1 and BcLAE1 are not interdependent.}

Previously, it was demonstrated that the full-length BcVEL1 fused to green fluorescent protein (GFP) is predominantly localized in the nuclei, while the BcVEL1 ${ }^{\mathrm{T} 4}$-GFP fusion protein is predominantly localized to the cytosol (Schumacher et al. 2012). To determine the distribution of BcVEL2 and BcLAE1, the other components of the proposed complex, corresponding GFP fusion proteins under the control of the constitutive oliC promoter of $A$. nidulans were expressed in different genetic backgrounds (discussed below) (Table 1). The subcellular localization of the three GFP fusion proteins indicated that BcVEL1 ${ }^{\mathrm{B} 05.10}$ and BcLAE1 predominantly and independently accumulate in the nuclei, while BcVEL2 is present in the cytosol and the nuclei (Fig. 1B). Based on these observations, it appears likely that the three proteins interact in the nuclei of B. cinerea $\mathrm{B} 05.10$.
BcLAE1 is dispensable under standard growth conditions.

To gain further insight into the signaling processes and downstream events of the VELVET complex in B. cinerea, deletion and overexpression mutants were functionally characterized. The generation of respective mutants for bcvell has been reported previously (Schumacher et al. 2012) (Table 1). The approach to replace bclael by a nourseothricin resistance cassette yielded two independent deletion mutants ( $\Delta$ bclae $1-T 3$, $\Delta$ bclael-T5). Complementation of the deletion mutant ( $\Delta$ bclae1-T3) was accomplished by reintroduction of bclael into the native gene locus yielding strains $\Delta b c l a e 1^{\mathrm{CIL}}$ (T1, T3). Expression of bclael under control of a constitutive promoter (A. nidulans PoliC) in the wild-type background resulted in three overexpressing mutants (OE::bclae1-T6, OE::bclae1-T7, OE::bclae1-T8). As the independent mutants exhibited identical phenotypes, the data for one arbitrarily chosen strain per mutation are presented (discussed below) (Table 1). Absence of the specific transcripts in $\Delta$ bcvell and $\Delta$ bclael mutants and the overexpression of the genes in OE::bcvell and OE::bclae1 mutants were confirmed by Northern blot analyses (Fig. 2A). Furthermore, these studies revealed that the expression levels of bcvel 2 and bcvel4 (ortholog of $A$. nidulans velC) were elevated in the $\Delta$ bcvell mutant and that bcvel 2 was slightly up-regulated in OE::bcvell and $\Delta$ bclael mutants.

Radial growth rates of the mutants and wild-type B05.10 on solid standard media, i.e., on Czapek-Dox (CD) as defined minimal medium and on complete medium (CM) under

A
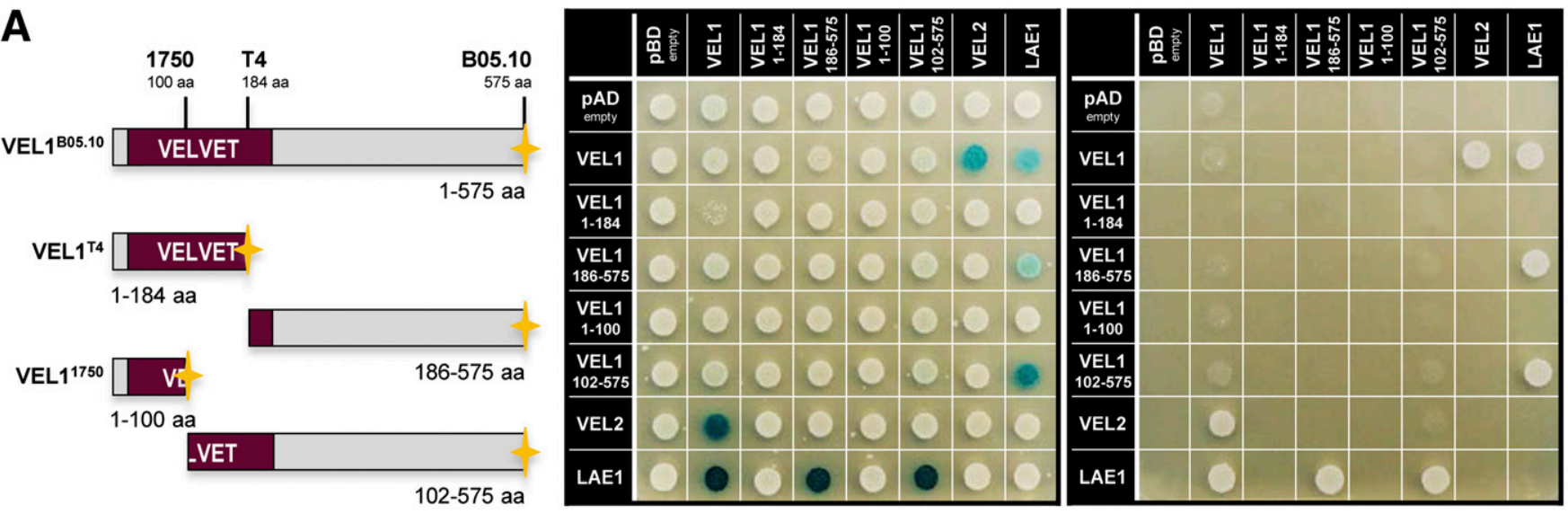

\section{B}
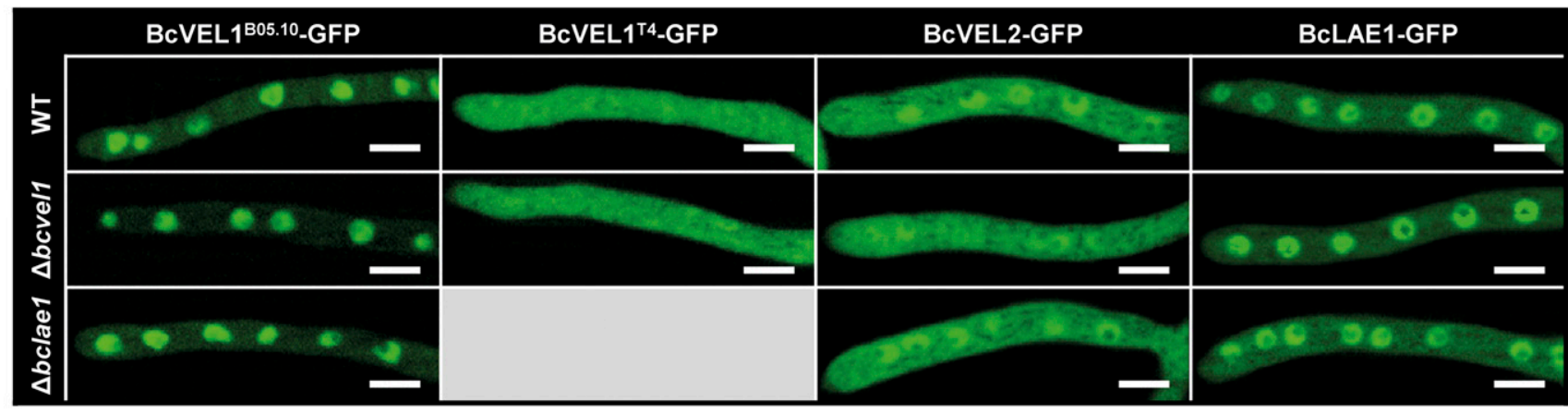

Fig. 1. BcVEL1, BcVEL2, and BcLAE1 form a complex in some but not all Botrytis cinerea strains. A, BcVEL1 ${ }^{\text {B05.10 }}$ but not the truncated proteins from T4 and 1750 interact with BcVEL2 and BcLAE1 in a yeast two-hybrid approach. The full-length proteins of BcVEL1, BcVEL2, and BcLAE1 found in strain B05.10, the truncated versions of BcVEL1 found in T4 and 1750 as well as the remaining C-terminal regions were fused to the GAL4 activation and GAL4 binding domains for yeast two-hybrid analysis. Cell suspensions of Saccharomyces cerevisiae strains (derivatives of PJ69-4A) containing the indicated vectors were dropped onto selective media. On the left: synthetic defined (SD) medium without leucine and tryptophan supplemented with X-Gal (SD-LW+X-Gal) was used to demonstrate the presence of both vectors and LacZ reporter gene activity (displayed by blue staining). On the right: SD without leucine, tryptophan, and histidine supplemented with $11 \mathrm{mM} 3$-amino-1,2,4-triazole (SD-LWH+3-AT) was used to detect HIS3 reporter gene activity (displayed by colony growth). B, Members of the VELVET complex are localized in the nuclei. Green fluorescent fusion proteins were expressed from the constitutive Aspergillus nidulans oliC promoter in different genetic backgrounds, i.e., in wild-type B05.10, $\Delta$ bcvell and $\Delta$ bclael. Conidia were incubated on microscope slides for $14 \mathrm{~h}$ in darkness, followed by $6 \mathrm{~h}$ in light. Scale bars, $5 \mu \mathrm{m}$. 
different light conditions (12-h light and 12-h dark photoperiod [LD] or constant darkness [DD]) were comparable (Table 2), demonstrating that the mutations of bclael and bcvell did not impair growth under these conditions. To monitor whether the $\Delta$ bclael mutants exhibit increased nitrate reductase activities under noninducing or repressing conditions, as previously reported for $\Delta b c v e l 1$ mutants (Schumacher et al. 2012), the strains were cultivated on solid CM supplemented with $\mathrm{KClO}_{3}$ (Table 2). While radial growth rates of the wild type were not significantly affected (91\% of control), indicating low expression levels of bcniaD, growth rates of both deletion mutants were decreased to a similar extent (approximately $75 \%$ of control), suggesting increased expression levels of bcniaD, leading to the more efficient conversion of chlorate to the toxic metabolite chlorite.

\section{BcLAE1 regulates OA formation and is required to cope with oxidative stress.}

Optimal growth rates for $B$. cinerea are achieved under acidic conditions ( $\mathrm{pH} 4$ to 5). Under neutral or alkaline conditions, the fungus first acidifies the culture medium by producing significant amounts of OA (Han et al. 2007) and, only then, it starts to colonize the medium. Growth rates of the mutant strains were not affected on standard media at $\mathrm{pH}$ values of 5 . However, changing the initial $\mathrm{pH}$ of the medium impaired the radial growth rates of the mutants. At $\mathrm{pH} 3$, growth rates of $\Delta b c l a e 1$, $\mathrm{OE}:$ bclae 1, and $\Delta$ bcvel1 mutants were affected; at $\mathrm{pH} 7$ and $\mathrm{pH}$ 9, those of $\Delta$ bclae1, $\Delta$ bcvel1, and $\mathrm{OE}:$ :bcvell mutants were affected (Fig. 2B). Reduced growth rates of the two deletion mutants under neutral or alkaline conditions was accompanied by the inability of the mutants to acidify the culture medium (Fig. 2C) and correlated with the absence of the expression of bcoahA encoding the OA-forming enzyme (Fig. 2A).

To assess whether either BcLAE1, BcVEL1, or both are also essential to cope with other kinds of stresses, growth rates of the mutants were quantified on media supplemented with stressors inducing osmotic stress $(0.7 \mathrm{M} \mathrm{NaCl}, 1.4 \mathrm{M}$ sorbitol) or oxidative stress (5.0 or $7.5 \mathrm{mM} \mathrm{H} \mathrm{H}_{2} \mathrm{O}_{2}, 500 \mu \mathrm{M}$ menadione) (Table 2). Under osmotic stress conditions all mutants showed comparable growth rates. However, the mutants had problems coping with oxidative stress conditions, especially during incubation in the light (LD). Hence, the addition of menadione (artificial source of superoxide radicals) decreased the growth rates of bclael and bcvell mutants. The addition of $\mathrm{H}_{2} \mathrm{O}_{2}$ along with the exposure to light had an even more pronounced effect on some mutants. Thus, $\Delta$ bclael mutants failed to grow on medium with $7.5 \mathrm{mN} \mathrm{H}_{2} \mathrm{O}_{2}$ in LD and DD, while the $\Delta$ bcvell and $\mathrm{OE}:$ :bcvell mutants were able to grow on the same medium in DD but not in LD conditions.

Taken together, BcLAE1 and BcVEL1 are required for the production of $\mathrm{OA}$ and, thereby, for facilitating growth under alkaline conditions. Moreover, BcLAE1 and, to a lesser extent, BcVEL1 contribute to oxidative stress resistance.

\section{Deletion of bclae1 decouples differentiation programs from light control.}

In the majority of B. cinerea isolates, including B05.10, the mode of asexual reproduction is controlled by the light condition (Canessa et al. 2013; Epton and Richmond 1980). (Macro)conidia, the primary source of inoculum for host infections, are formed during exposure to light, while sclerotia, which serve as resting structures (asexual) and the female parent in sexual reproduction, are only produced in the absence of light. Time course experiments were performed to follow the onset of conidiation in the generated mutants under different illumination conditions (Fig. 3A). Both $\Delta$ bclael and $\Delta b c v e l 1$ mutants initiated conidiation earlier in LD and constant light (LL) conditions, relative to the wild type, and produced conidia even in DD conditions. In LL, growth and conidiation of $\Delta$ bclael, $\Delta$ bcvell, and OE::bcvell was further accompanied by increased production of the dark pigment DHN-melanin. On the other hand, the onset of conidiation was slightly delayed in OE::bclae1 mutants.

The formation of sclerotia requires a longer period than conidiation. Usually nonpigmented initials are visible after 1 week in DD. After 2 weeks, the sclerotia are fully developed and pigmented (Fig. 3B). While OE::bclae1 mutants formed sclerotia as the wild type, $\Delta$ bclae 1 and $\Delta$ bcvell mutants failed to form any sclerotia and the OE::bcvell mutant produced significant numbers of both conidia and sclerotia in DD. Though both $\Delta$ bclael and $\Delta$ bcvell mutants underwent conidiation in DD, their colony appearances were different. While $\Delta$ bcvell mutants sparingly developed aerial hyphae accompanied by high numbers of conidia, the $\Delta$ bclae 1 mutants exhibited excessive aerial hyphae formation, giving the colonies a 'cotton candy'-like appearance (data not shown), and produced only small numbers of conidia (Fig. 3B).

Due to the lack of sclerotia formation, both deletion mutants were female sterile. However, $\Delta$ bclael mutants produced

Table 1. Botrytis cinerea strains used in this study

\begin{tabular}{|c|c|c|}
\hline Strain & Genotype & Reference \\
\hline WT:B05.10 & Isolate from grapevine, $M A T 1-1$ & Quidde et al. 1998 \\
\hline WT:SAS405 & Isolate from grapevine, $M A T 1-2$ & Faretra et al. 1988 \\
\hline$\Delta$ bcvell & B05.10, sbcvell::hph; homokaryon & Schumacher et al. 2012 \\
\hline OE::bcvell & B05.10, PoliC::bcvel1-gfp::nat1 in bcniiA; heterokaryon & Schumacher et al. 2012 \\
\hline$\Delta$ bclae1 & B05.10, Abclae1::nat1; homokaryon & This study \\
\hline OE::bclae1 & B05.10, PoliC::bclae1::hph in bcniaD; heterokaryon & This study \\
\hline$\Delta$ bclae $1^{\mathrm{CiL}}$ & B05.10, $\Delta$ bclae1::nat1, bclae1::hph in loco; heterokaryon & This study \\
\hline WT::VEL1 ${ }^{\text {B05.10_GFP }}$ & B05.10, PoliC::bcvel1 ${ }^{\mathrm{B} 05.10}$-gfp::nat1 in bcniiA; heterokaryon & Schumacher et al. 2012 \\
\hline WT::VEL1 ${ }^{\text {T4_GFP }}$ & B05.10, PoliC::bcvel1 ${ }^{\mathrm{T} 4}$-gfp::nat1 in bcniiA; heterokaryon & Schumacher et al. 2012 \\
\hline WT::VEL2-GFP & B05.10, PoliC::bcvel2-gfp::hph in bcniiA; heterokaryon & This study \\
\hline WT::LAE1-GFP & B05.10, PoliC::bclael-gfp::hph in bcniiA; heterokaryon & This study \\
\hline$\Delta$ bcvel1::VEL1 ${ }^{\text {B05.10_GFP }}$ & B05.10, $\Delta$ bcvel1 ::hph, PoliC::bcvel1 ${ }^{\mathrm{B} 05.10}$-gfp::nat1 in bcniiA; heterokaryon & Schumacher et al. 2012 \\
\hline$\Delta$ bcvel1::VEL1 ${ }^{\mathrm{T} 4}$-GFP & B05.10, $\Delta$ bcvell ::hph, PoliC::bcvel1 ${ }^{\mathrm{T} 4}$-gfp::nat1 in bcniiA; heterokaryon & Schumacher et al. 2012 \\
\hline$\Delta$ bcvell::VEL2-GFP & B05.10, $\Delta$ bcvell ::hph, PoliC::bcvel2-gfp::nat1 in bcniiA; heterokaryon & This study \\
\hline$\Delta$ bcvel1::LAE1-GFP & B05.10, $\Delta$ bcvel1::hph, PoliC::bclae1-gfp::nat1 in bcniiA; heterokaryon & This study \\
\hline Dbclae1::VEL1 ${ }^{\mathrm{B} 05.10}$-GFP & B05.10, Dbclae1::nat1, PoliC::bcvel1 ${ }^{\mathrm{B} 05.10}-$ gfp::hph in bcniiA; heterokaryon & This study \\
\hline Dbclae1::VEL2-GFP & B05.10, $\Delta$ bclae1::nat1, PoliC::bcvel2-gfp::hph in bcniiA; heterokaryon & This study \\
\hline$\Delta$ bclae1::LAE1-GFP & B05.10, $\Delta$ bclae1::nat1, PoliC::bclae1-gfp::hph in bcniiA; heterokaryon & This study \\
\hline$\Delta b c w c l 1$ & B05.10, $\Delta b c w c l 1:: h p h ;$ homokaryon & Canessa et al. 2013 \\
\hline$\Delta b c f l u G$ & B05.10, $\Delta b c f l u G:: h p h ;$ homokaryon & This study \\
\hline OE::bcfluG & B05.10, PoliC::bcfluG::nat1 in bcniaD; heterokaryon & This study \\
\hline
\end{tabular}


microconidia as the wild type (data not shown) and, consequently, the $\Delta$ bclae 1 microconidia were used to fertilize sclerotia of the reference strain SAS405, carrying the opposite mating type. As previously reported for the cross SAS405 ${ }^{\text {sclerotia }} \times \Delta$ bcvell ${ }^{\text {microconidia }}$ (Schumacher et al. 2012), the cross SAS $405^{\text {sclerotia }} \times \Delta b$ clae $1^{\text {microconidia }}$ yielded regularly shaped apothecia (fruiting bodies) with fertile ascospores. Of 47 ascospores tested, 24 yielded nourseothricin-sensitive colonies with the wild-type phenotype (sclerotia in DD) and 23 yielded nourseothricin-resistant colonies exhibiting the $\Delta$ bclael phenotype (no sclerotia but conidia in DD) (data not shown), demonstrating that $\Delta$ bclael mutants were male fertile.

\section{Bclae1 and bcvel1 deletion mutants still respond to light on the transcriptional level.}

Bcvell and bclael deletion mutants do not appropriately respond to environmental light conditions as the asexual differentiation programs are deregulated. Recently, genome-wide expression analyses in strain B05.10 revealed a number of genes whose expression levels are up- or down-regulated in response to white light (Schumacher et al. 2014). Two TF were described to be important for appropriate light signaling: the ortholog of WHITE COLLAR-1, BcWCL1 (Canessa et al. 2013), and the light-responsive transcription factor BcLTF1 (Schumacher et al. 2014).

To gain insight into the role of the VELVET complex in the light signaling network, Northern blot analyses were performed to detect the expression levels of several known light-responsive genes encoding TF (bcwcl1, bcwcl2, bcltf1, bcltf2, bcltf3) and photoreceptors (bccry1, bccry2, bop1, bcphy2). For this, wild type, $\Delta$ bcvell, $\Delta$ bclael, as well as the partially 'blind' $\Delta b c w c l 1$ mutant (Canessa et al. 2013) were cultivated for 2 days on solid $\mathrm{CM}$ with cellophane overlays in DD or LL. Moreover, DD-grown cultures were exposed to white light for 15, 60, and $300 \mathrm{~min}$ (Fig. 4). Surprisingly, the expression levels of the tested genes increased similarly to the wild type in response to light in the $\Delta b c v e l 1$ and $\Delta$ bclael mutants, while induction of gene expression in the $\Delta b c w c l 1$ mutant did not occur or was less evident. Nevertheless, minor effects were observed for some genes in the mutant backgrounds. Hence, bccry2, encoding one of the near-UV and blue light-sensing cryptochromes, was more strongly up-regulated in $\Delta$ bcvell and $\Delta$ bclae 1 mutants. Furthermore, bcltfl and bcltf 2 were already expressed in $\Delta b c v e l l$ mutants in the dark and were more strongly induced, compared with the wild type, upon $15 \mathrm{~min}$ of light exposure. In conclusion, $\Delta b c v e l 1$ and $\Delta$ bclael mutants still respond to light signals with altered gene expression patterns, though the conidiation program no longer depends on light.

The FluG ortholog has no obvious functions in $\mathbf{B}$. cinerea.

Gene expression studies revealed that the expression levels of the gene BC1G_01105/B0510_5527 (hereafter called bcfluG), encoding the ortholog of the $A$. nidulans conidiation-related protein FluG (Lee and Adams 1994), were significantly upregulated in mutants exhibiting a hyper-conidiation phenotype, such as in $\Delta b c l t f 1$ mutants (Schumacher et al. 2014) and in $\Delta$ bcvell and $\Delta$ bclael mutants (Fig. 4). Considering the possibility that the FluG ortholog is involved in conidiation in $B$. cinerea as well, bcfluG deletion and overexpressing mutants were generated (discussed below) and were characterized with regard to conidiation as well as to sclerotia formation, mating, apothecial development, and virulence (Supplementary Fig. S2) (data not shown). However, in all these assays the $b c f l u G$ mutants exhibited the same phenotype as the wild type.

\section{BcLAE1 is essential for colonizing the host tissue.}

$B$. cinerea is able to enter intact plant tissues by using two different kinds of penetration structures, conidia that form short germ tubes that penetrate immediately and hyphae that differentiate highly branched infection cushions (Choquer et al. 2007). As mutants may be affected in either one or both modes of infection, primary leaves of French bean (Phaseolus vulgaris) plants were inoculated with conidial suspensions (Fig. 5A) and undifferentiated mycelia of wild-type and mutant strains (data not shown). Infections by the overexpression mutants proceeded in a wild type-like fashion when conidial suspensions were used as inoculum. However, when mycelium of the OE::
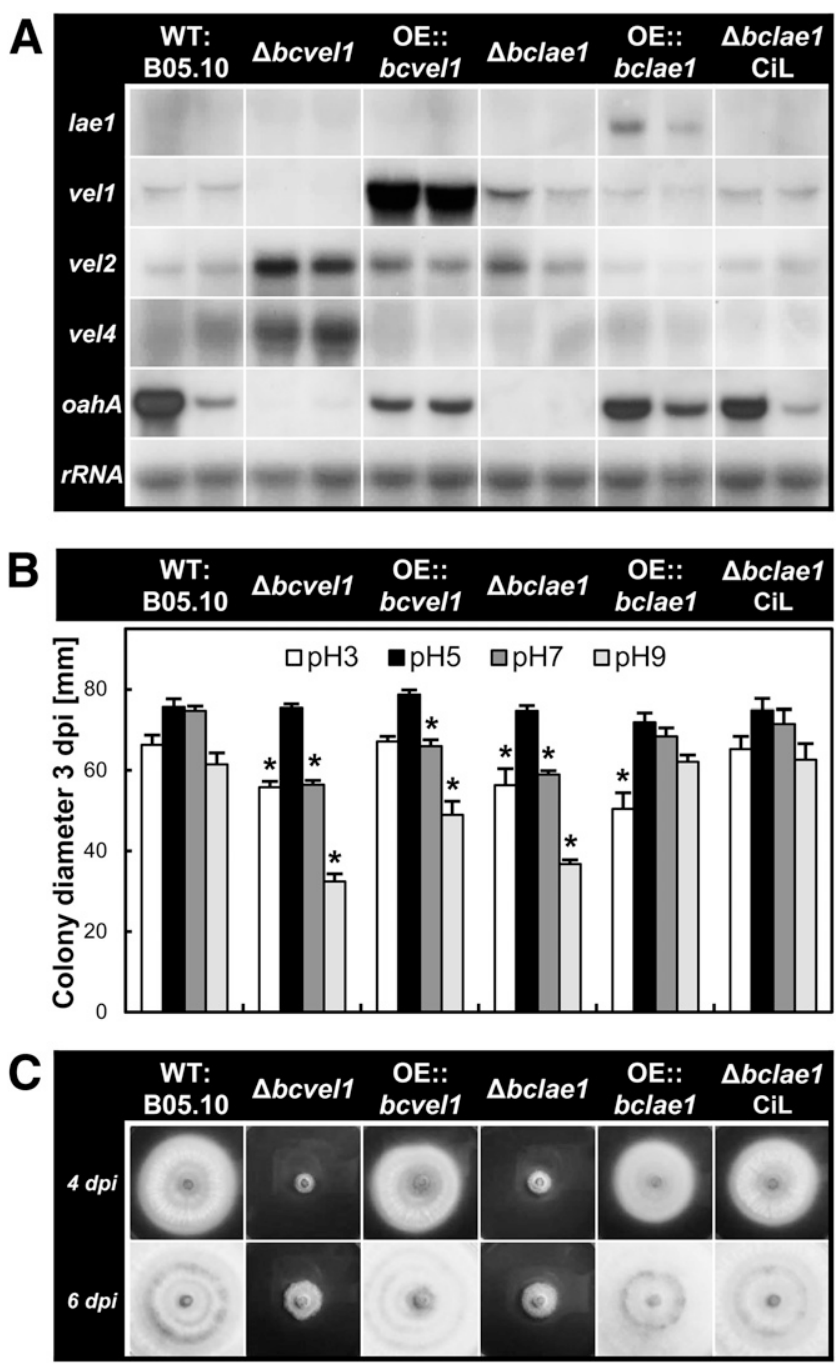

Fig. 2. BcVEL1 and BcLAE1 are required for production of oxalic acid $(\mathrm{OA})$ and, thereby, for facilitating growth under neutral and alkaline conditions. A, Northern blot analyses confirm the deletion and overexpression of bcvell and bclae 1 in respective mutants. Indicated strains were cultivated for 2 (left lanes) and 3 days (right lanes) on solid complete medium (CM) (pH 5) covered with cellophane overlays in a 12-h light and12-h dark (LD) photoperiod. The blot was hybridized with probes of bclae1, bcvell to bcvel4 (no expression was detectable for bcvel3), and bcoahA encoding the OA-forming enzyme oxaloacetate hydrolase. The 28S rRNA is shown as loading control. B, Mutations of BcVEL1 and BcLAE1 impair the ability to tolerate extreme $\mathrm{pH}$ values. Strains were incubated for 3 days on solid $\mathrm{CM}$ adjusted to the indicated $\mathrm{pH}$ values. Mean values and standard deviations were calculated from three colonies per strain and condition. Asterisks indicate significant differences compared with WT:B05.10 in each condition $(P<0.001)$. C, Bcvell and bclael deletion mutants fail to acidify the culture medium. For monitoring the acidification of the medium, solid CM, $\mathrm{pH} 8$, was supplemented with the $\mathrm{pH}$ indicator bromothymolblue. Strains were cultivated for 6 days in a LD photoperiod. The bright color indicates acidification $(\mathrm{pH}<6.0)$. 
bclael strain was used, infections were irregular, which was likely caused by reduced penetration efficiencies rather than by impaired invasive growth rates (data not shown). Penetration and the establishment of primary lesions by $\Delta$ bclael and $\Delta$ bcvell were similar to the wild type, but then, infection proceeded differentially. Both mutants were impaired in their capabilities to colonize the surrounding host tissue. However, while conidia-derived infections did not spread at all, infections starting from mycelial plugs spread slowly and finally stopped in the stage of spreading (data not shown). In any case, the deletion mutants failed to colonize the whole leaf and to produce conidia, indicating that both BcLAE1 and BcVEL1 are required for full virulence. Similar defects in colonization and in planta conidiation were observed when ripe grape berries (Vitis vinifera) were inoculated with conidial suspensions (Fig. 5B). While $92 \%$ of berries were fully colonized by the wildtype strain at 10 days postinoculation, only 71 and $54 \%$ of the berries infected with the $\Delta$ bclael and $\Delta$ bcvell mutants, respectively, were similarly colonized.

\section{BcLAE1 and BcVEL1 have common}

\section{and unique target genes.}

To gain broader insight into the functions of BcLAE1 and BcVEL1 and their interrelationship, a genome-wide approach was initiated to compare gene expression profiles of wild-type B05.10 and $\Delta$ bcvell and $\Delta$ bclael mutants. Conidia of the strains were cultivated for $48 \mathrm{~h}$ on solid grape juice medium covered with cellophane overlays. Total RNA from four biological replicates was extracted, labeled, and hybridized to NimbleGen microarrays containing oligonucleotides representing all predicted genes of B. cinerea (Amselem et al. 2011). Statistical tests led to the identification of 1,438 distinct genes whose expression levels were significantly altered in one or both $\Delta$ bclael and $\Delta$ bcvell mutants (fold change [FC[ $>2$, $P$ value $<0.05$ ) (discussed below). These differentially expressed (DE) genes were assigned to eight expression profiles (Fig. 6A; Supplementary Table S2). 36.5\% (525) of the DE genes show the same expression pattern in both mutants, with $14 \%$ (202) being overexpressed (profile 1) and $22.5 \%$ (323) being underexpressed (profile 4). A total of 29.2\% (420) are overexpressed in only one mutant, $13.4 \%$ (193) in $\Delta$ bcvell (profile 2) and $15.8 \%$ (227) in $\Delta$ bclael (profile 3). A total of
$32.1 \%$ (462) are underexpressed in only one mutant, $10.1 \%$ (146) in $\Delta$ bcvell (profile 5) and $22 \%$ (316) in $\Delta$ bclael (profile 6). Only $2.2 \%$ (31 genes) exhibit opposing expression patterns in the two mutant backgrounds (profiles 7 and 8).

Functional enrichment analyses using the GSEA (gene set enrichment analyses) method with defined sets of annotated genes were performed to reveal biological processes that are affected by the absence of one or both BcVEL1 and BcLAE1 (Table 3). These analyses revealed that the groups of underexpressed genes in both deletion mutants were enriched for genes belonging to SM clusters as well as for genes encoding secreted proteins, proteases, and carbohydrate-active enzymes. Furthermore, the group of underexpressed genes in $\Delta$ bclael was enriched for genes encoding enzymes involved in oxidative stress response (OSR), and the group of overexpressed genes in $\Delta$ bclael was enriched for genes encoding transporters for various substrates.

Taken together, the microarray analyses demonstrated that $\mathrm{BcVEL} 1$ and BcLAE1 regulate a set of common target genes but that the majority of DE genes $(63.5 \%)$ is differently affected by the absence of BcVEL1 and BcLAE1. Nevertheless, both components of the VELVET complex are required for the appropriate expression of genes involved in SM and cell-wall degradation.

\section{Absence of one or both BcLAE1 and BcVEL1 affects SM-related gene expression.}

The transcriptomic approach revealed that 16 of the 44 genes encoding SM KE were differentially expressed relative to wild type in either $\Delta$ bcvell, $\Delta$ bclae 1, or both mutants (Fig. 6B). Only one KE-encoding gene (bcstc4) was significantly overexpressed in both mutants (FC of 4.6 and 4.1), and two others (bcpks5, encoding a hybrid PKS-NRPS, and bcnrps7) were significantly overexpressed in the $\Delta b c v e l l$ mutant (FC of 3 and 3.5) and to a lesser extent in the $\Delta$ bclael mutant (FC of 1.9 and 1.5) Bcpks5 and bcnrps 7 are colocalized in the genome together with other genes that were overexpressed in $\Delta$ bcvell, suggesting that the two KE-encoding genes belong to the same gene cluster (B0510_10357/bcnrps 7 to B0510_10348/bcpks5).

Ten KE-encoding genes were underexpressed in both mutants to similar extents (profile 4). Only bcpks19 and bcdmat2,

Table 2. Radial growth rates of wild type B05.10 and mutant strains ${ }^{\mathrm{a}}$

\begin{tabular}{|c|c|c|c|c|c|c|c|}
\hline \multicolumn{2}{|c|}{ Medium/light condition } & \multirow{2}{*}{$\frac{\text { WT:B05.10 }}{52.8 \pm 3.7(70 \%)}$} & \multirow{2}{*}{$\frac{\text { sbcvell }}{42.3 \pm 0.8(56 \%)}$} & \multirow{2}{*}{$\frac{\text { OE::bcvell }}{\mathrm{n} / \mathrm{a}}$} & \multirow{2}{*}{$\frac{\text { sbclae1 }}{45.2 \pm 3.0(60 \%)}$} & \multirow{2}{*}{$\frac{\text { OE::bclae1 }}{\text { n/a }}$} & \multirow{2}{*}{$\frac{\text { sbclae1 }^{\text {CiL }}}{50.0 \pm 3.5(67 \%)}$} \\
\hline CD-NO3 & LD & & & & & & \\
\hline pH5 & DD & $54.2 \pm 2.6(70 \%)$ & $43.3 \pm 1.0(57 \%)$ & $\mathrm{n} / \mathrm{a}$ & $49.8 \pm 1.0(66 \%)$ & $\mathrm{n} / \mathrm{a}$ & $56.0 \pm 1.3(73 \%)$ \\
\hline CD-NH4 & LD & $45.2 \pm 2.4(60 \%)$ & $46.5 \pm 4.8(62 \%)$ & $53.2 \pm 2.6(68 \%)$ & $49.0 \pm 2.0(66 \%)$ & $36.3 \pm 4.1(50 \%)$ & $39.8 \pm 2.2(53 \%)$ \\
\hline pH5 & DD & $50.0 \pm 1.7(65 \%)$ & $48.7 \pm 1.6(64 \%)$ & $55.0 \pm 2.4(70 \%)$ & $52.5 \pm 1.2(70 \%)$ & $43.5 \pm 4.7(58 \%)$ & $50.3 \pm 1.9(66 \%)$ \\
\hline CM pH5 & LD & $75.7 \pm 1.9(100 \%)$ & $75.4 \pm 1.0(100 \%)$ & $78.7 \pm 1.2(100 \%)$ & $74.8 \pm 1.2(100 \%)$ & $72.0 \pm 2.3(100 \%)$ & $74.9 \pm 2.8(100 \%)$ \\
\hline Control & DD & $77.1 \pm 2.8(100 \%)$ & $75.8 \pm 1.1(100 \%)$ & $78.2 \pm 1.4(100 \%)$ & $75.2 \pm 1.5(100 \%)$ & $74.5 \pm 1.5(100 \%)$ & $76.5 \pm 3.3(100 \%)$ \\
\hline $\mathrm{CM}+$ & LD & $43.3 \pm 3.6(57 \%)$ & $42.8 \pm 0.8(57 \%)$ & $36.8 \pm 2.3(47 \%)$ & $41.5 \pm 0.5(56 \%)$ & $42.3 \pm 3.4(59 \%)$ & $45.7 \pm 1.2(61 \%)$ \\
\hline 0.7 M NaCl & DD & $45.2 \pm 0.8(59 \%)$ & $40.8 \pm 1.6(54 \%)$ & $35.7 \pm 0.5(46 \%)$ & $39.0 \pm 1.8(52 \%)$ & $40.3 \pm 2.3(54 \%)$ & $45.0 \pm 1.4(59 \%)$ \\
\hline $\mathbf{C M +}$ & LD & $65.2 \pm 0.8(86 \%)$ & $64.0 \pm 0.6(85 \%)$ & $62.5 \pm 1.2(79 \%)$ & $57.3 \pm 0.5(77 \%)$ & $65.0 \pm 0.6(90 \%)$ & $65.8 \pm 0.8(88 \%)$ \\
\hline 1.4 M sorbitol & DD & $60.8 \pm 1.5(79 \%)$ & $60.5 \pm 1.4(80 \%)$ & $60.2 \pm 0.4(77 \%)$ & $57.3 \pm 2.1(76 \%)$ & $58.8 \pm 1.2(79 \%)$ & $62.8 \pm 1.3(82 \%)$ \\
\hline CM+ & LD & $69.7 \pm 1.9(92 \%)$ & $58.0 \pm 1.5(77 \%)$ & $\mathrm{n} / \mathrm{a}$ & $56.0 \pm 1.7(75 \%)$ & $\mathrm{n} / \mathrm{a}$ & $61.2 \pm 5.7(82 \%)$ \\
\hline $0.4 \mathrm{M} \mathrm{KClO}_{3}$ & DD & $70.2 \pm 1.7(91 \%)$ & $54.3 \pm 1.0(72 \%)$ & $\mathrm{n} / \mathrm{a}$ & $56.5 \pm 0.5(75 \%)$ & $\mathrm{n} / \mathrm{a}$ & $65.5 \pm 1.6(86 \%)$ \\
\hline $\mathbf{C M +}$ & LD & $45.0 \pm 4.3(59 \%)$ & $15.5 \pm 12.5(20 \%)$ & $36.0 \pm 12.3(48 \%)$ & $8.0 \pm 6.4(11 \%)$ & $45.7 \pm 3.5(63 \%)$ & $49.3 \pm 2.7(66 \%)$ \\
\hline $5.0 \mathrm{mM} \mathrm{H} \mathrm{H}_{2} \mathrm{O}_{2}$ & DD & $51.5 \pm 0.8(67 \%)$ & $38.5 \pm 3.6(49 \%)$ & $43.3 \pm 1.9(57 \%)$ & $32.7 \pm 6.1(43 \%)$ & $46.2 \pm 3.8(62 \%)$ & $53.2 \pm 0.4(69 \%)$ \\
\hline $\mathrm{CM}+$ & LD & $40.1 \pm 4.5(53 \%)$ & $0.0 \pm 0.0(0 \%)$ & $4.5 \pm 7.0(6 \%)$ & $0.0 \pm 0.0(0 \%)$ & $36.8 \pm 2.9(51 \%)$ & $40.5 \pm 6.9(54 \%)$ \\
\hline $7.5 \mathrm{mM} \mathrm{H}_{2} \mathrm{O}_{2}$ & DD & $40.5 \pm 2.4(53 \%)$ & $31.2 \pm 7.5(41 \%)$ & $34.0 \pm 2.5(43 \%)$ & $0.0 \pm 0.0(0 \%)$ & $37.2 \pm 0.8(50 \%)$ & $40.8 \pm 0.8(53 \%)$ \\
\hline $\mathrm{CM}+500 \mu \mathrm{M}$ & LD & $52.8 \pm 0.8(70 \%)$ & $38.7 \pm 1.0(51 \%)$ & $42.0 \pm 0.6(53 \%)$ & $42.2 \pm 0.8(56 \%)$ & $40.5 \pm 3.9(56 \%)$ & $50.2 \pm 0.4(67 \%)$ \\
\hline menadione & DD & $52.3 \pm 1.5(68 \%)$ & $32.3 \pm 1.0(43 \%)$ & $45.8 \pm 0.8(59 \%)$ & $42.7 \pm 2.1(57 \%)$ & $50.2 \pm 0.8(67 \%)$ & $51.0 \pm 2.4(67 \%)$ \\
\hline
\end{tabular}

a Strains were cultivated for three days on minimal medium $(\mathrm{CD})$ containing nitrate $\left(\mathrm{NO}_{3}\right)$ or ammonium $\left(\mathrm{NH}_{4}\right)$ as the nitrogen source or complete medium (CM) supplemented with stressors as indicated. Cultures were incubated in a 12-h light and12-h dark (LD) photoperiod and in constant darkness (DD). Mean values and standard deviations were calculated from three colonies per strain and condition, with two measurements per colony. Relative growth to control $(\mathrm{CM}, \mathrm{pH} 5)$ is indicated in brackets; $\mathrm{n} / \mathrm{a}=$ not analyzed. Respective mutants are affected in nitrate assimilation due to targeted integration of the overexpression (OE) constructs at bcniiA (OE::bcvell) and bcniaD (OE::bclae1), respectively. 
putatively encoding a PKS and a DMATS, respectively, seem to be less affected in the absence of bclael (FC of -2.9 and -2.4 ) than by the absence of bcvell (FC of -7.2 and -7.7). bcpaxl, which is possibly responsible for the biosynthesis of an indolediterpene such as paxilline, belongs to the KE-encoding genes that were underexpressed in both mutants in a similar fashion (FC of -3.9 and -3.1). Notably, the surrounding genes (B0510_1781 to B0510_1785) that are also orthologous to those in the paxilline biosynthetic gene cluster in other fungi (Saikia et al. 2008) exhibit the same expression pattern. The PKSencoding genes bcboa6 and bcboa9 that are involved in BOA biosynthesis (Dalmais et al. 2011) were also underexpressed in both mutants. All BOA cluster genes except bcboal4 to bcboal7 showed the same expression pattern as the two KE. Furthermore, the KE-encoding genes bcpks4 and bcnrps 8 were underexpressed in both mutants together with their adjacent genes, indicating that they belong to a yet-undescribed cluster
(B0510_7880 to B0510_7886). Finally, three other independent PKS-encoding genes, including the putative melanogenic gene bcpks12 (Schumacher et al. 2014), were less expressed in the absence of either BcVEL1 or BcLAE1.

In contrast, $b c p k s 7$, encoding a hybrid PKS-NRPS, and the adjacent genes (B0510_5671, B0510_5672) were significantly underexpressed in the $\Delta b c$ vell mutant only $(\mathrm{FC}-3.6)$ and not affected by the deletion of bclae1. In contrast, bcbot 1 and four adjacent genes (bcbot2-5) involved in the biosynthesis of BOT (Pinedo et al. 2008; Siewers et al. 2005) as well as bcnrps 1 and three adjacent genes (B0510_8343, B0510_8342, and B0510_8340) were severely affected by the deletion of bclae1 but not significantly by $\Delta b c v e l 1$.

To investigate whether the overexpression of bcvell and bclael affects the expression levels of chosen SM-related genes belonging to profiles 1 (bcstc4), 4 (bcboa6), 5 (bcpks7), and 6 (bcbot 2 ), wild type and deletion and overexpressing mutants for

A

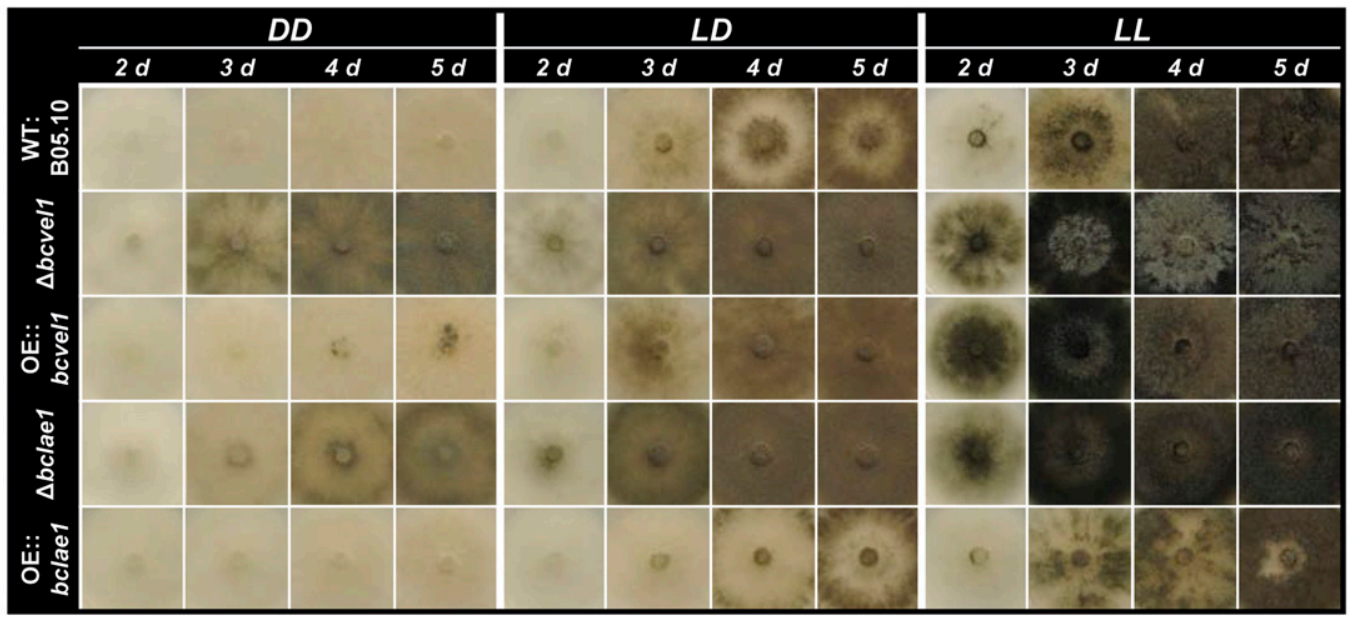

B
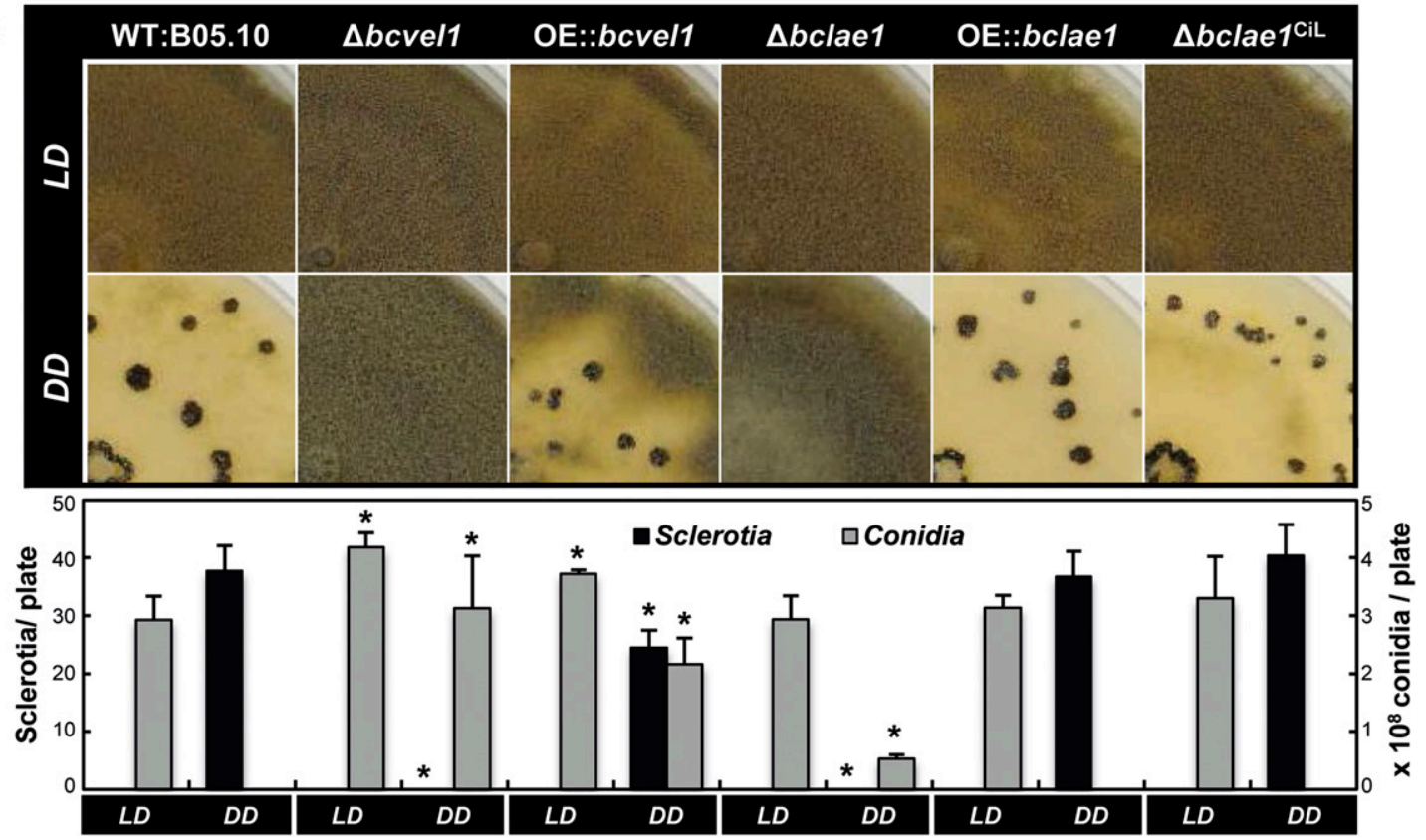

Fig. 3. Deletions of $b c v e l 1$ and bclae 1 decouple differentiation programs from light control. A, Conidiation starts earlier in $\Delta b c v e l 1$ and $\Delta b c l a e 1$ mutants than in the wild type and occurs in constant darkness. Indicated strains were cultivated on solid complete medium (CM) (petri dishes with a diameter of 45 mm) in continuous darkness (DD), in a 12-h light and 12-h dark (LD) photoperiod, or continuous light (LL). Plates were inoculated with plugs of undifferentiated mycelia on consecutive days; pictures were taken after 5 days of incubation. B, Light-independent conidiation of $\Delta b c v e l 1$ and $\Delta b c l a e 1$ mutants is accompanied by the loss of sclerotia formation. Strains were incubated for 14 days on solid CM (petri dishes with a diameter of 90 mm) in DD or LD conditions. The numbers of formed sclerotia and conidia were determined from three cultures per strain and light condition. Asterisks indicate significant differences compared with WT:B05.10 in each condition $(P<0.001)$. 
bcvell and bclael were cultivated on solid grape juice medium as described above. Isolated RNA was used for quantitative reverse transcription-polymerase chain reaction (RT-PCR) analyses that confirmed the expression patterns obtained for the deletion mutants in the microarray approach, except for bcbot 2 , whose reduced expression levels in $\Delta b c v e l 1$ is significant in the quantitative (q)PCR data while it was not in the microarray data (Fig. 6C). However, the overexpression of bcvell and bclael did not or only marginally affected the expression levels of the chosen genes.

In sum, the expression studies provide evidence that BcVEL1 and BcLAE1 are important regulators of SM-related gene expression in B. cinerea. Thereby, the coregulation patterns of known clusters (BOA, BOT) were confirmed and five cryptic new gene clusters (bcpks5/bcnrps 7, bcpks4, bcpax 1, bcpks 7 , and bcnrps1) were identified, based on the coregulation of the KE-flanking genes by one or both BcVEL1 and BcLAE1.

\section{DISCUSSION}

Recently, we identified genetic variation in bcvell, the ortholog of $A$. nidulans veA, as the genetic basis for reduced virulence, loss of $\mathrm{OA}$ formation, and the hyper-conidiation phenotype of wild-type strain T4 (Schumacher et al. 2012). Bcvell ${ }^{\mathrm{T} 4}$ contains a SNP (compared with strain B05.10) leading to a truncated protein of 184 aa. Consequently, replacement of this allele by bcvell ${ }^{\mathrm{B} 05.10}$ rescued virulence, sclerotia formation, and OA secretion in the T4 genetic background. A second SNP resulting in a truncated protein of 100 aa was identified in the BIK-producing strain 1750 (Schumacher et al. 2013). As both strains still exhibit regular pigmentation in contrast to the generated deletion mutants lacking the whole gene, we suggest that the first 100 aa of BcVEL1 are sufficient for proper regulation of pigment formation but not for regulation of differentiation, $\mathrm{OA}$ formation, and virulence. The full-length protein $\left(\mathrm{BcVEL} 1^{\mathrm{B} 05.10}\right.$ ) accumulates in the nuclei, whereas the truncated protein $\left(\mathrm{BcVEL}^{\mathrm{T} 4}\right)$ predominantly localizes to the cytosol. Therefore, we concluded that the $\mathrm{C}$-terminus and its putative interaction partners are essential to regulate the phenotypic traits virulence, differentiation, and OA formation.

To further characterize the regulatory networks of BcVEL1, we studied the role of its putative interaction partner, the putative methyltransferase BcLAE1. Yeast two-hybrid analyses confirmed that the $B$. cinerea orthologs (BcVEL1, BcLAE1, BcVEL2) interact and likely form a complex analogous to the situation found in eurotiomycetes and sordariomycetes (Bayram et al. 2008; Hoff et al. 2010; Wiemann et al. 2010). Thereby, $\mathrm{BcVEL}^{\mathrm{B} 05.10}$ —unlike BcVEL1 ${ }^{\mathrm{T} 4}$ and BcVEL1 ${ }^{1750}$-interacts with BcLAE1 and BcVEL2. The interaction with BcLAE1 is mediated via the C-terminal region (186 to 575 aa), while interaction with BcVEL2 probably requires the intact $\mathrm{N}$-terminal VELVET domain. This hypothesis is corroborated by the data of Yang et al. (2013), who demonstrated by a yeast-two hybrid approach that the truncated BcVEL1 protein comprising the first 294 aa, and thereby, the whole VELVET domain (aa 23 to 220), interacts with BcVEL2 (truncated protein containing aa 107 to 451). The latter finding differs from the situation in A. nidulans, in which the first 29 aa of VeA facilitate the interaction with VelB, whereas the VELVET domain itself is dispensable (Bayram et al. 2008; Palmer et al. 2013). The first 36 aa of $\mathrm{VeA}$ also determine the subcellular localization of the protein (Stinnett et al. 2007). In contrast to VeA, BcVEL1 lacks an obvious nuclear localization signal (NLS), though the full-length protein localizes to the nucleus in the wild type as well as in the $\triangle$ bclae1 mutant, indicating that BcLAE1 is not required for nuclear localization of full-length BcVEL1. Furthermore, absence of BcVEL1 does not alter the subcellular localization

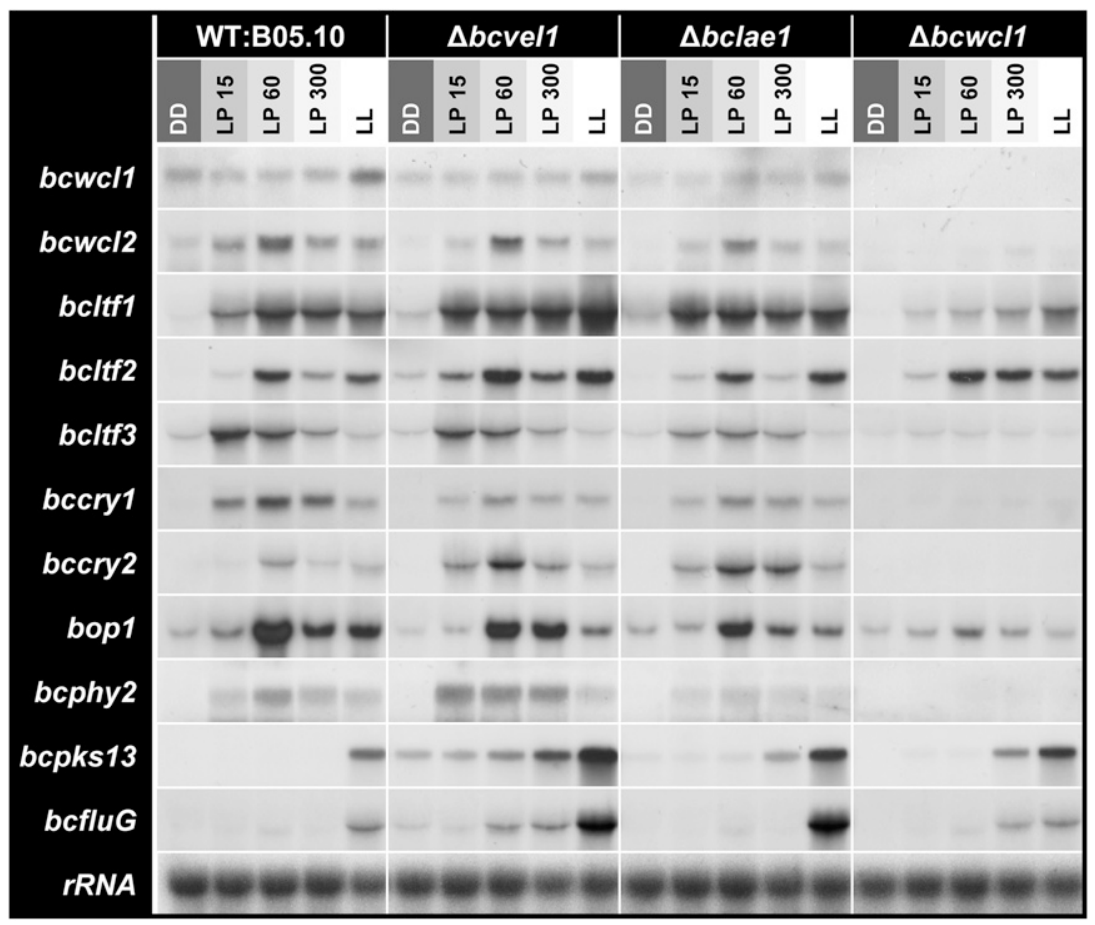

Fig. 4. $\Delta$ bcvell and $\Delta$ bclael mutants still respond to light on the transcriptional level. Wild-type B05.10 and indicated mutants were incubated on solid complete medium for $50 \mathrm{~h}$ in continuous darkness (DD) or continuous light (LL) conditions. DD-grown cultures were then exposed to white light for the indicated periods (light pulses [LP] of 15, 60, and $300 \mathrm{~min}$ ) or were kept for an additional 60 min in DD before harvest. The Northern blot was hybridized with the following probes: $b c w c l 1, b c w c l 2$ (transcription factors forming the WHITE COLLAR complex), $b c l t f 1$ to $b c l t f 3$ (light-responsive transcription factors), bccry1, bccry2 (putative UV light-sensing cryptochromes), bopl (opsin-like protein), bcphy2 (putative red light-sensing phytochrome), bcpks13 (polyketide synthase, putatively involved in melanin biosynthesis), and bcfluG (ortholog of Aspergillus nidulans FluG). The 28S rRNA is shown as loading control. 
patterns of BcLAE1 (without NLS) and BcVEL2 (with NLS) that are predominantly found in the nucleus and in the cytosol and nucleus, respectively. However, the complex likely comprises further proteins that may determine the nuclear localization of BcVEL1 and BcLAE1, as demonstrated in A. nidulans (Bayram et al. 2008; Park et al. 2014; Purschwitz et al. 2008). Additional interaction partners in this system include VosA (interacting with VeA and LaeA), VelC (interacting with VosA), and the phytochrome FphA, that interacts also with the blue light-sensing WHITE COLLAR complex (LreA/LreB). The observation that bcvel4, encoding the VelC ortholog, is overexpressed in the $\Delta b c v e l l$ background in a similar fashion as bcvel2 and bclae1 may indicate an interrelationship with the BcVEL1BcLAE1 complex. However, bcvel3, encoding the VosA ortho$\log$ that was recently shown to function as a TF of developmental regulatory genes (Ahmed et al. 2013), was not expressed in any of the studied conditions (data not shown).

The physical interactions of BcVEL1-BcVEL2 and BcVEL1-BcLAE1 suggest that the proteins are functionally interdependent and that the lack of any component prevents the formation of the complex and subsequent signaling while the single overexpression of a complex member is not sufficient to amplify the signaling responses, as OE::bcvel1 and OE::bclae1 mutants did not exhibit opposing phenotypes relative to their respective deletion strains. Remarkably, the overexpression of bcvell resulted in an intermediate phenotype that is more similar to those of the deletion mutants than to that of the wild type, suggesting the possibility that the stability of the proteinprotein complex is affected in order to avoid inadequate signaling. In agreement with the expected functional interdependency of the complex members, the deletions of bcvell and bcvel 2 in strain 38B1 resulted in similar phenotypes (Yang et al. 2013), and deletions of bcvell and bclael in the B05.10 background (this study) resulted in similar, though not identical, phenotypes, i.e., similarly reduced virulence on French bean and grape, loss of OA accumulation, and decoupling of the differentiation programs from light control. Further mutants have been described that exhibit the 'blind' phenotype characterized by persistent conidiation. Among them are two mutants lacking the TF involved in light sensing and signal transduction, BcWCL1 as a blue-light sensor (Canessa et al. 2013), and BcLTF1 as a lightresponsive TF that modulates the expression levels of other lightresponsive genes (Schumacher et al. 2014). However, unlike the $\Delta$ bcwcll mutant, $\Delta b c v e l 1$ and $\Delta$ bclael mutants are still able to
A

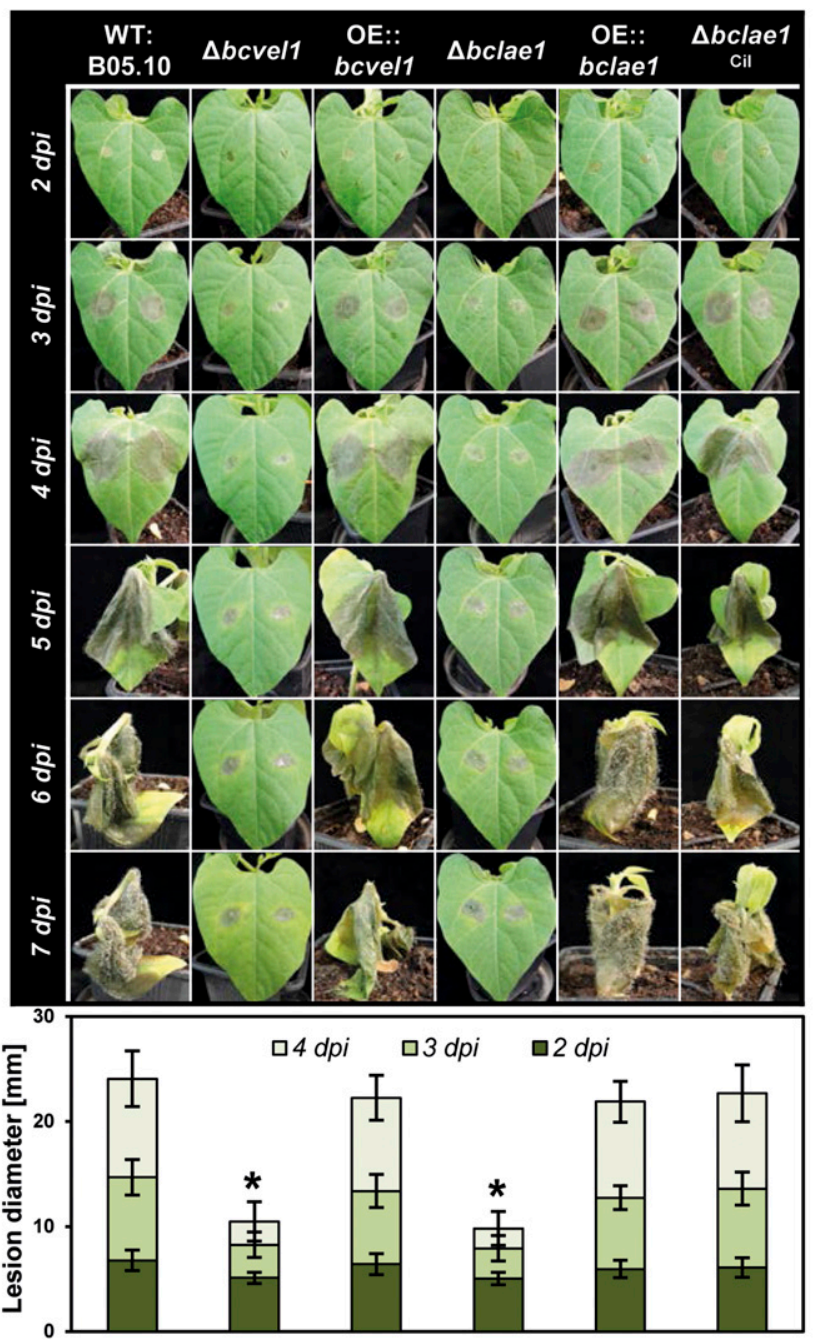

B

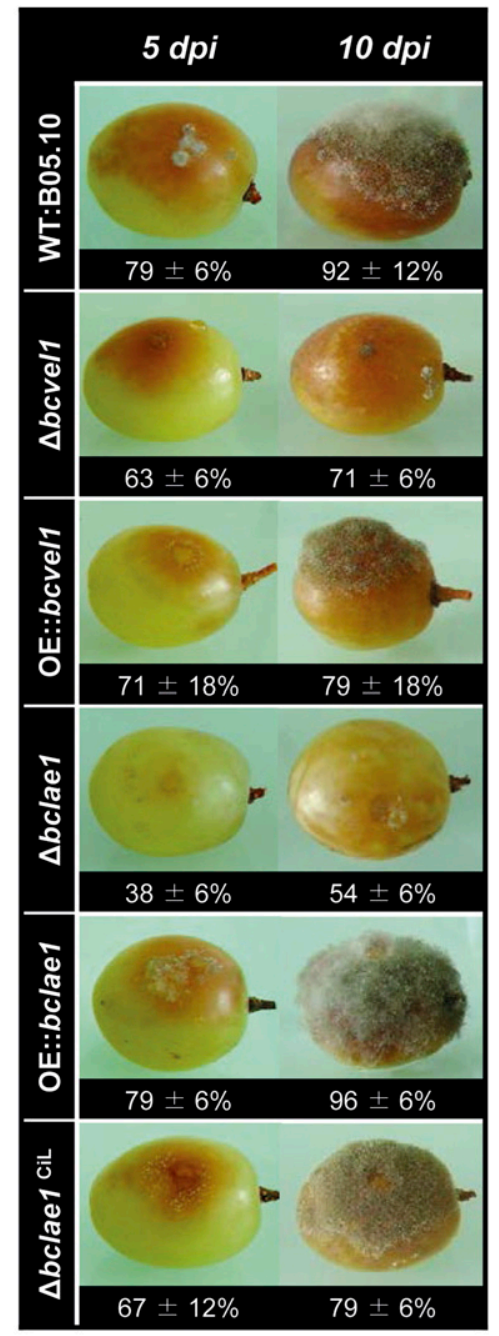

Fig. 5. BcVEL1 and BcLAE1 are essential for necrotrophic growth. A, Bcvell and bclael deletion mutants are impaired in colonization of French bean leaves. Primary leaves of living Phaseolus vulgaris plants were inoculated with conidial suspensions and incubated in a 12-h light and 12-h dark (LD) photoperiod. Mean values and standard deviation were calculated from 24 lesions per strain with two measurements per lesion. Asterisks indicate significant differences compared with WT:B05.10 in each condition $(P<0.001)$. B, Bcvell and bclael deletion mutants are impaired in colonization of grape berries. Berries were inoculated with conidial suspensions and incubated in LD conditions. The most representative berries for each strain are shown, while the numbers below indicate the percentage of colonized berries from two independent assays with 12 berries per strain. 
sense light and to turn on light-responsive gene expression. Hence, it appears likely that BcVEL1 and BcLAE1 in addition to BcWCL1 and BcLTF1 act as repressors of conidiation by affecting a yet-unknown TF responsible for switching on the particular differentiation program. This role is fulfilled by the light-responsive TF, i.e., BrlA in A. nidulans (Adams et al. 1988; Ruger-Herreros et al. 2011) and by Fl ('fluffy') in Neurospora crassa (Bailey and Ebbole 1998; Olmedo et al. 2010). The genome of B. cinerea, however, lacks orthologs of either BrlA or Fl (Amselem et al. 2011). Approaches are currently in progress to identify the TF accomplishing the initiation of conidiation in B. cinerea.

$B c f l u G$ encoding a glutamine synthetase (GS)-like protein and the ortholog of A. nidulans FluG was identified as an overexpressed gene in $\Delta b c l t f l$ (Schumacher et al. 2014). Its expression pattern mirrors that of bcpks 13, encoding the PKS
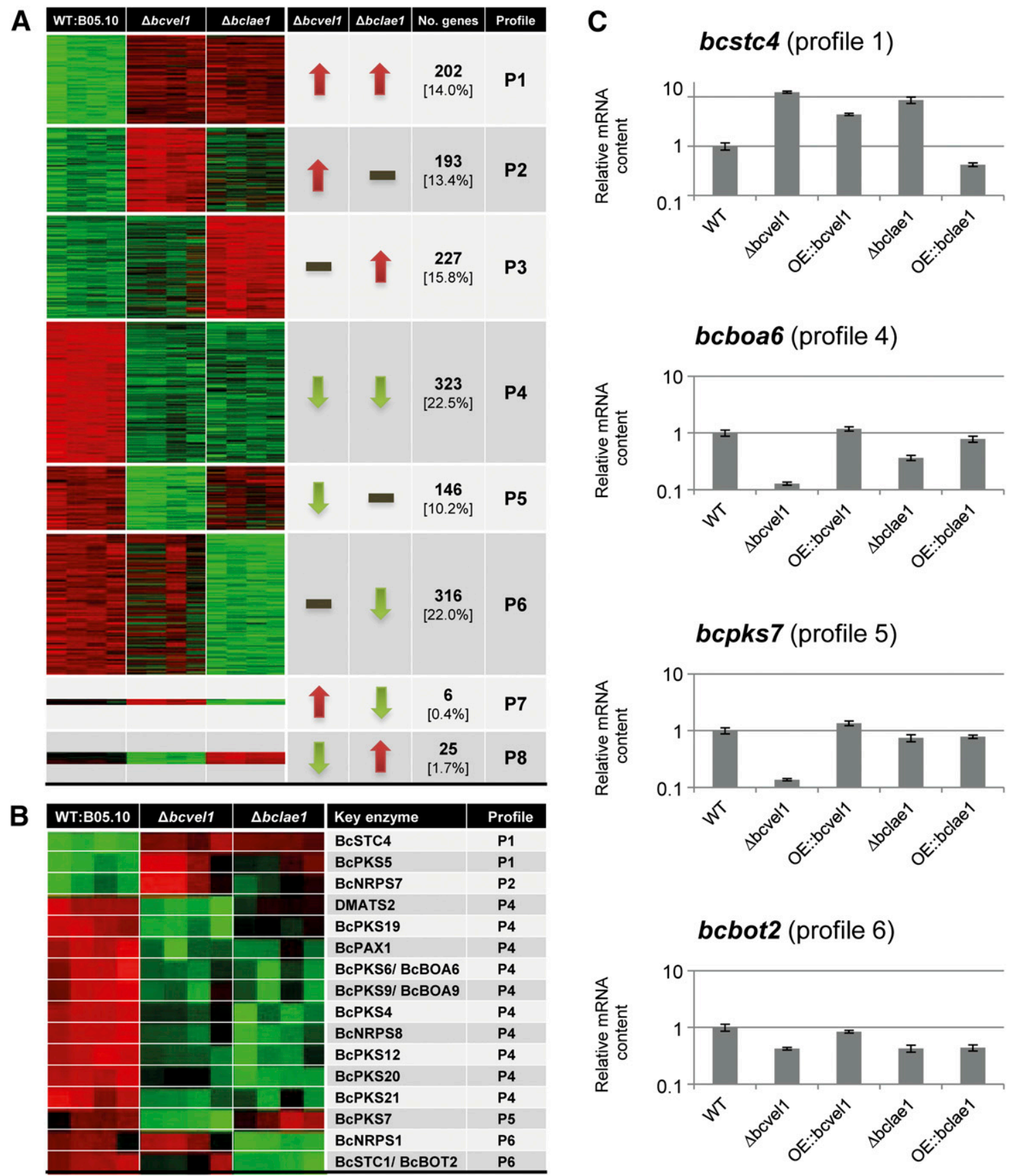

\section{bcpks7 (profile 5)}

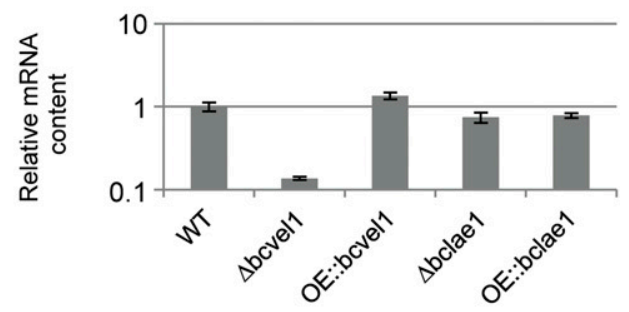

\section{bcbot2 (profile 6)}

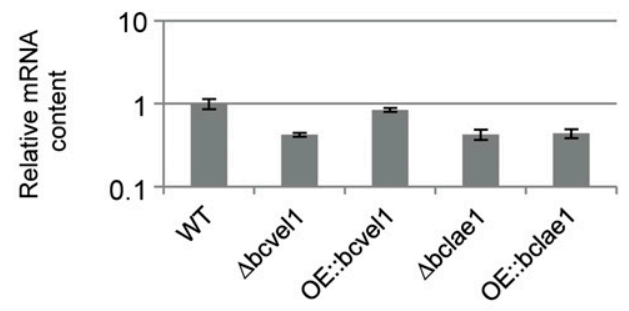

Fig. 6. Deletions of bcvell and bclael affect the expression levels of 1,438 genes. Conidial suspensions of wild-type B05.10 and mutant strains $\Delta b c v e l 1$ and $\Delta$ bclael were spread onto solid grape juice medium with cellophane overlays and were incubated for $48 \mathrm{~h}$. RNA from four biological replicates was isolated, labeled, and hybridized to microarrays containing all predicted genes of Botrytis cinerea. A and B, Relative expression values of the genes (i.e., log2normalized intensities scaled by gene) in the three strains (twelve hybridizations) were clustered and depicted by color scale, in which shades of green and red represent under- and overexpressed genes, respectively. A, Profiles of the differentially expressed genes. Statistical analysis revealed 1,438 genes whose expression levels are affected in either $\Delta$ bcvell (profiles P2, P5), $\Delta$ bclae1 (profiles P3, P6) or in both mutants (profiles P1, P4, P7, P8). B, Sixteen of 44 secondary metabolite key enzyme (KE)-encoding genes are differentially expressed in one or both $\Delta b c v e l 1$ or $\Delta b c l a e 1$. C, Quantitative reverse transcriptionpolymerase chain reaction results confirm the expression patterns of four chosen KE-encoding genes in either $\Delta b c v e l 1, \Delta b c l a e 1$, or both and indicate that these genes do not show opposite expression patterns in the overexpression mutants (OE::bcvell and OE::bclae1). Gene expression levels were calculated following the $2^{-\Delta \Delta \mathrm{CT}}$ method using constitutively expressed bcactl (actin) as a reference and the wild-type strain B05.10 as a control. 
likely involved in melanin biosynthesis and with the onset of conidiation in the wild type and the $\Delta b c v e l l$ mutant in this study. Therefore, we assumed a role of $b c f l u G$ in regulation of conidiation according to the model in A. nidulans, in which FluG forms a small, secreted molecule that triggers conidiation once it has been sensed by a membrane-spanning receptor. Accordingly, loss-of-function mutants do not form conidia ('fluffy') (D'Souza et al. 2001; Lee and Adams 1994; Yager et al. 1998). Furthermore, a role of FluG in negative regulation of sclerotial development has been reported in A. flavus. In this system, no diffusible SM was detected; however, the authors demonstrated that FluG interacts via the GS domain with VelB and LaeA and suggested that FluG may exercise control of conidiation and sclerotia formation via the VELVET complex (Chang et al. 2012, 2013). However, the FluG ortholog does not exert obvious functions in $B$. cinerea, as neither deletion nor overexpression of $b c f l u G$ affects light-dependent differentiation programs (formation of conidia, sclerotia, apothecia), virulence, or stress responses.

BcLAE1 and, to a lesser extent, BcVEL1 are involved in the oxidative stress response. Hence, deletion mutants are more sensitive to $\mathrm{H}_{2} \mathrm{O}_{2}$ and menadione and the growth defects become more evident during cultivation in the light. In recent studies, the additive effect of light and $\mathrm{H}_{2} \mathrm{O}_{2}$ treatment on oxidative stress levels has been described in B. cinerea (Schumacher et al. 2014). In these studies, $\Delta b c l t f 1$ mutants lost the ability to tolerate light or oxidative stress (in darkness). As light tolerance is restored by antioxidants (Schumacher et al. 2014), it was concluded that light provokes oxidative stress and that, accordingly, the OSR system, including genes for detoxification of ROS, is required during exposure to light. In accordance with the more pronounced effect of $\mathrm{H}_{2} \mathrm{O}_{2}$ on radial growth rates of $\Delta$ bclael mutants compared with those of the $\Delta b c v e l 1$ mutants, the group of underexpressed genes in $\Delta b c l a e 1$ is enriched for genes encoding OSR enzymes. Nevertheless, light (LD) alone does not impair growth of $\Delta b c v e l 1$ and $\Delta b c l a e 1$ mutants on nutrient-poor or -rich media. Increased sensitivity of deletion mutants to oxidative stress has been reported in other fungal systems as well, e.g., for C. heterostrophus, $\Delta v e l 1$ and $\Delta$ lae1 (Wu et al. 2012), for $M$. oryzae, $\Delta v e A$ and $\Delta v e l B$ (Kim et al. 2014), and T. atroviride, $\Delta$ lael mutants (Aghcheh et al. 2013).

BcVEL1 and BcLAE1 are essential for virulence by facilitating the colonization of the plant tissue. Notably, the penetration via germ tubes or infection cushions is not affected in the deletion mutants, leading to the establishment of primary infections that are indistinguishable from those provoked by the wild type. However, lesions of the mutants do not spread, suggesting that the mutants are not able to kill surrounding host cells. The reason for this effect is difficult to predict, and it appears unlikely that just one factor is responsible. Hence, OA,
BOT, BOA, unknown SM, and a number of secreted enzymes (proteases and glycosyl hydrolases) may contribute to virulence. Especially striking were the significantly decreased expression levels of the latter genes in both mutants, usually highly expressed during the fungus-host interaction. Similarly, the loss of mycoparasitic activity of T. atroviride $\Delta$ lael mutants correlates with the underexpression of several genes normally induced during the mycoparasitic interaction, e.g., proteases, glucanases, and small secreted proteins (Aghcheh et al. 2013).

The transcriptomics approach for comparing BcVEL1- and BcLAE1-dependent gene expression pattern was performed by using young, conidia-derived cultures of the wild type and the deletion mutants. The growth medium made from concentrated grape juice was previously shown to be well-suited to induce SM-related genes, such as the BOA and BOT genes, and also genes for which usually no or very low expression levels were found under axenic conditions (Simon et al. 2013) (data not shown). The comparative approach revealed that BcVEL1 and BcLAE1 have common and distinct target genes. Sixteen genes encoding SM-KE were differentially affected in at least one deletion mutant. To date, only two gene clusters (BOA, BOT) have been linked to the corresponding metabolites. The concomitant reduced production of these two toxins under some growth conditions (here grape juice medium) could contribute to the reduced virulence of the bclael and bcvell mutants, as it is the case for the previously characterized bcbot $2 / b c b o a 6$ double mutants (Dalmais et al. 2011). Another KE-encoding gene underexpressed in both mutants is bcpks12, which was recently considered as a candidate gene for melanin synthesis during sclerotial development (Schumacher et al. 2014). Deregulation of this gene could be linked to the fact that the mutants do not form sclerotia. The expression of the alternative melanogenic gene, bcpks13, that is likely responsible for conidial pigmentation was not affected in the mutants, but this is probably due to growth conditions. Indeed, the 48-h-old mycelia grown on grape juice medium was almost white. In contrast, during growth on solid CM medium in constant light, BcVEL1 and BcLAE1 repress bcpks13 expression as previously described for BcVEL1 (Schumacher et al. 2012, 2013).

Furthermore, the microarray approach allowed for identifying the borders of further clusters of coregulated genes. Most of these new B. cinerea gene clusters (bcpks5/bcnrps 7, bcpks4/ bcnrps8, bcpks7) or isolated KE-encoding genes (bcstc4, bcpks19, bcpks20) are not present in the genome of $S$. sclerotiorum nor in other fungal species, suggesting that B. cinerea produces many specific compounds that remain to be identified. Some of the gene clusters dependent on one or both BcVEL1 and BcLAE1 (BOA, bcpks5/bcnrps7, bcnrps1, bcpks12) include a TF-encoding gene that may contribute to the specific regulation of the adjacent genes. Moreover, under the

Table 3. Gene set enrichment analysis (GSEA) of $\Delta b c v e l 1$ - and $\Delta b c l a e 1$-dependent genes ${ }^{\mathrm{a}}$

\begin{tabular}{|c|c|c|c|}
\hline \multirow[b]{3}{*}{ Name of gene set (size) } & \multicolumn{3}{|c|}{ Normalized enrichment score } \\
\hline & \multirow{2}{*}{$\frac{\Delta b c v e l 1}{\text { Underexpressed }}$} & \multicolumn{2}{|c|}{$\Delta$ bclae1 } \\
\hline & & Underexpressed & Overexpressed \\
\hline Secondary metabolism gene clusters (289) & $1.90\left(<10^{-3}\right)$ & $2.02\left(<10^{-3}\right)$ & $\mathrm{n} / \mathrm{s}$ \\
\hline Secondary metabolism key enzymes (44) & $1.66\left(<10^{-3}\right)$ & $1.65\left(<10^{-3}\right)$ & $\mathrm{n} / \mathrm{s}$ \\
\hline Early secretome (227) & $1.85\left(<10^{-3}\right)$ & $1.97\left(<10^{-3}\right)$ & $\mathrm{n} / \mathrm{s}$ \\
\hline Carbohydrate-active enzymes (388) & $1.61\left(<10^{-3}\right)$ & $1.63\left(<10^{-3}\right)$ & $\mathrm{n} / \mathrm{s}$ \\
\hline Proteases (377) & $1.59\left(<10^{-3}\right)$ & $1.41\left(<10^{-3}\right)$ & $\mathrm{n} / \mathrm{s}$ \\
\hline Oxidative stress response (OSR) (103) & $\mathrm{n} / \mathrm{s}$ & $1.84\left(<10^{-3}\right)$ & $\mathrm{n} / \mathrm{s}$ \\
\hline Transporters (393) & $\mathrm{n} / \mathrm{s}$ & $\mathrm{n} / \mathrm{s}$ & $1.56\left(<10^{-3}\right)$ \\
\hline
\end{tabular}

${ }^{a}$ Gene sets were previously defined by Amselem et al. (2011), Espino et al. (2010), and Schumacher et al. (2014). The GSEA toolkit was used to determine whether gene sets show statistically significant concordant differences due to deletion of either bcvell or bclae 1 . Normalized enrichment score and $P$ values (in brackets) are indicated for cases where $P$ values $<0.05 ; \mathrm{n} / \mathrm{s}=$ not specified, $P$ value $>0.05$. 
same growth conditions (grape juice medium), several of the clusters were also shown to be dependent on one or both BcLTF1 (data not shown) and BcYOH1, a C2H2-TF that acts as a global transcriptional regulator (Simon et al. 2013). Therefore, metabolite production by $B$. cinerea depends, as in other fungal systems (Brakhage 2013), on several global regulatory proteins, including those of the VELVET complex and globally active TF, and pathway-specific TF.

In conclusion, this study provides evidence for overlapping and distinct roles of BcVEL1 and BcLAE1 in light-dependent differentiation and virulence of the gray mold fungus. The loss of both genes decouples conidiation from light control that is accompanied by the absence of sclerotial development. Due to the lack of sclerotia that act as female-parent in sexual reproduction, both deletion mutants are female-sterile, but they are able to reproduce sexually via acting as the male parent. Both BcVEL1 and BcLAE1 have a strong impact on the expression of SM-related genes including those involved in phytotoxin biosynthesis and on secreted enzymes and are therefore required for necrotrophic development.

\section{MATERIALS AND METHODS}

\section{Cultivation of $B$. cinerea strains.}

Strain B05.10 of Botrytis cinerea Pers.: Fr. is an isolate from Vitis vinifera, its genome sequence is available (Broad Institute database) (Amselem et al. 2011; Staats and van Kan 2012) and is used in this study as the recipient strain for genetic modifications (Table 1). Strains were cultivated in petri dishes containing solid synthetic CM (Pontecorvo et al. 1953) or modified $\mathrm{CD}$ as minimal medium $\left(2 \%\right.$ sucrose, $0.1 \% \mathrm{KH}_{2} \mathrm{PO}_{4}$, $0.05 \% \mathrm{KCl}, 0.05 \% \mathrm{MgSO}_{4} \cdot 7 \mathrm{H}_{2} \mathrm{O}, \mathrm{pH} 5.0$ ) containing either $0.3 \% \mathrm{NaNO}_{3}(\mathrm{CD}-\mathrm{NO} 3)$ or $0.1 \%\left(\mathrm{NH}_{4}\right)_{2} \mathrm{SO}_{4}(\mathrm{CD}-\mathrm{NH} 4)$ as nitrogen source. Acidification of the culture medium (solid $\mathrm{CM}, \mathrm{pH} 8$ ) was monitored using the $\mathrm{pH}$ indicator bromothymol blue $(0.1 \%)$ (Sigma-Aldrich). Cultures were incubated at $20^{\circ} \mathrm{C}$ under white light (LD or LL) for conidiation, and in continuous darkness for induction of sclerotial formation. White light (9000 l× at culture level) was generated by using a set of Sylvania Standard F18W/29-530 'warm white' and F36W/33-640 'cool white' fluorescent bulbs. Crosses using SAS405 (MAT1-2) as reference strain were performed according to Faretra and Antonacci (1987). For DNA and RNA isolation, mycelia were grown on solid CM medium with cellophane overlays.

\section{Standard molecular methods.}

Fungal genomic DNA was prepared according to Cenis (1992). Total RNA was isolated, making use of the TRIzol reagent (Invitrogen). For Northern blot analyses, samples $(25 \mu \mathrm{g})$ of total RNA were transferred to Hybond-N+ membranes after electrophoresis on a $1 \%(\mathrm{wt} / \mathrm{vol})$ agarose gel containing formaldehyde, according to the method of Sambrook et al. (1989). For Southern blot analysis, fungal genomic DNA was digested with restriction enzymes (Fermentas), was separated on $1 \%$ (wt/vol) agarose gels, and was transferred to Amersham Hybond-N+ filters (GE Healthcare Ltd.). Blot hybridizations with randomprimed $\alpha-{ }^{32} \mathrm{P}-\mathrm{dCTP}$-labeled probes were performed as described previously (Siewers et al. 2004). PCR reactions were performed using the high-fidelity DNA polymerase Phusion (Finnzymes) for cloning purposes and the BioTherm Taq DNA Polymerase (GeneCraft) for diagnostic applications. All primers that have been used in this study for cloning, diagnostic PCR analyses, sequencing, and qRT-PCR analyses are listed in Supplementary Table S1. Replacement fragments and expression vectors were assembled in Saccharomyces cerevisiae by exploiting its homologous recombination machinery (Colot et al. 2006; Schumacher 2012). Sequencing of DNA fragments was performed with the
Big Dye Terminator v3.1 sequencing kit (Applied Biosystems) in an ABI Prism capillary sequencer (model 3730; Applied Biosystems). For sequence analysis, the software package DNAStar was used. Protocols for protoplast formation and transformation of B. cinerea were described previously (Schumacher 2012). Resistant colonies were transferred to solid CM supplemented with hygromycin B (Invitrogen) or nourseothricin (WernerBioagents) in a concentration of $70 \mu \mathrm{g} / \mathrm{ml}$.

\section{Construction of bclae1 mutants.}

For generation of a bclael replacement fragment, the 5' $(0.845 \mathrm{~kb})$ and $3^{\prime}$ noncoding regions $(1.017 \mathrm{~kb})$ of bclael were amplified using primers bclae $1-5 \mathrm{~F}$ and bclae 1-5R and bclae 1 $3 \mathrm{~F}$ and bclae 1-3R and were assembled with a nourseothricin resistance (natR) cassette (PtrpC::natl, amplified with primers $h p h-\mathrm{F}$ and $h p h \mathrm{R}-\operatorname{trp} C$-T2, using pZPnat 1 as template) by yeast recombinational cloning, yielding plasmid $\mathrm{p} \Delta$ bclae 1 -natR. The replacement fragment $(2.974 \mathrm{~kb})$ was amplified with primers bclae $1-5 \mathrm{~F}$ and bclae $1-3 \mathrm{R}$ and was transformed into $B$. cinerea B05.10. Homologous integration events in nourseothricinresistant transformants were detected by diagnostic PCR using the primers natl-hiF and trpC-P binding in the natR cassette, and the primers bclael-HI-F and bclael-HI-R binding upstream and downstream of the bclael-flanking regions, respectively. Single spore isolates were screened for the absence of bclae1 alleles using primers bclae1-F2 and bclae1-R2, matching the substituted coding region. For Southern blot analysis, genomic DNA of the mutants ( $\Delta$ bclae 1-T3 and $\Delta$ bclae1-T5) and the recipient strain B05.10 was digested with $E c o$ RV, and the blot was hybridized with the $5^{\prime}$ flank of the replacement fragment. The loss of a restriction site within bclael results in larger hybridizing fragments in the replacement mutants $(4.4 \mathrm{~kb})$ than in the wild type $(1.6 \mathrm{~kb})$. Complementation of the deletion mutant $\Delta$ bclael-T3 was accomplished by targeted integration of bclael at the native gene locus, resulting in the replacement of the natR cassette. The vector $\mathrm{p} \Delta$ bclae $\mathrm{I}^{\mathrm{CIL}}$-hygR was generated in yeast by cotransformation of pRS426/EcoRI+XhoI, the 5'-flank + bclae1 (primers bclae 1-5F and bclae1-Tgluc-R), Tgluc (primers Tgluc-F2 and $\mathrm{T} g l u c$-natR), the hygromycin resistance cassette (PtrpC:: $h p h$ ) from pCSN44 (primers $h p h-\mathrm{F}$ and $h p h-\mathrm{R}$ ), and the bclael 3'-flank (primers bclae1-3F and bclae1-3R). Targeted integration of the construct and, thereby, the restoration of the $5^{\prime}$ region of bclael was verified in two hygromycin-resistant transformants $\left(\Delta\right.$ bclae $^{\mathrm{CIL}}-\mathrm{T} 1, \Delta$ bclae $\left.^{\mathrm{CIL}}-\mathrm{T} 3\right)$ by using primer combinations bclae1-hi5F and bclael-R4 and bclae1-hi3R and $h p h$-hiR (data not shown). For overexpressing bclae1, the ORF was amplified with primers bclae 1-PoliC-F and bclae1-Tgluc-R and was assembled with the NcoI+NotI-digested plasmid pNDH-OGG (Schumacher 2012) in yeast, yielding pNDHPoliC::bclae1. The vector contains sequences for targeted integration at bcniaD (locus of the nitrate reductase), a hygR cassette, and the expression cassette with bclael under control of the constitutive oliC promoter from A. nidulans. The construct was transformed into B05.10. Targeted integration at bcniaD-5' was detected in hygromycin-resistant transformants OE::bclae1-T6, OE::bclae1-T7, and OE::bclae1-T8 by diagnostic PCR using primers bcniaD-hi5F and Tgluc-hiF (data not shown). Overexpression of bclael in the respective transformants was confirmed by Northern blot analyses (Fig. 2A) (data not shown).

\section{Localization studies.}

To determine the subcellular localization of BcVEL1, BcVEL2, and BcLAE1, the genes were fused to $g f p$ and were expressed in different genomic backgrounds, i.e., in WT: B05.10, $\Delta$ bcvel1-T22, and $\Delta$ bclae1-T3 (Table 1). For vector 
construction, the genes were amplified using primers with overlaps to PoliC and Tgluc, and amplicons were assembled with NcoI-digested plasmids pNAH-OGG or pNAN-OGG (Schumacher 2012) containing a hygR and a natR cassette, respectively. Targeted integration of the constructs at bcniiA was confirmed by diagnostic PCR, using primer combinations bcniiA-hi5F and Tgluc-hiF, bcniiA-hi3R and hph-hiF, or bcniiA-hi3R and nat1-hiF (data not shown). For microscopy, conidia of two transformants per fusion construct were suspended in Gamborg B5 solution supplemented with $2 \%$ glucose and $0.02 \%$ ammonium phosphate and were incubated overnight on microscope slides. Nuclei were stained using the fluorescent dye Hoechst 33342 as described previously (Schumacher 2012). Fluorescence and light microscopy was performed with a Zeiss AxioImager.M1 microscope equipped with the ApoTome.2 technology for optical sectioning with structured illumination. Differential interference microscopy was used for bright-field images. Specimens stained by Hoechst 33342 were examined using the filter set 49 4',6-diamidino-2-phenylindole shift free (excitation G 365, beam splitter FT 395, emission BP 445/50) and GFP fluorescence using filter set 38 (excitation BP 470/40, beam splitter FT 495, emission BP 525/50). Images were captured with an AxioCam MRm camera and were analyzed using the Axiovision Rel 4.8 software package (Zeiss). GFP signals in the generated mutants colocalized with the Hoechst staining patterns, indicating that BcVEL1 ${ }^{\mathrm{B} 05.10}$, BcVEL2, and BcLAE1 accumulated in the nuclei in the different genetic backgrounds, i.e., in wild-type B05.10, $\Delta$ bcvell, and $\Delta$ bclael (data not shown). Expression of BcLAE1-GFP in the $\Delta$ bclael background restored the formation of sclerotia in the dark and the acidification of the culture medium (data not shown), demonstrating the functionality of the BcLAE1-GFP fusion protein.

\section{Construction of bcfluG mutants.}

The replacement construct $\Delta b c f l u G$-hygR was generated by integrating the $5^{\prime}(1.425 \mathrm{~kb})$ and $3^{\prime}$ noncoding $(0.357 \mathrm{~kb})$ regions of $b c f l u G$ and the hygR cassette from pCSN44 (PtrpC:: $h p h)$ in pRS426. The replacement fragment $(3.218 \mathrm{~kb})$ was amplified by PCR, using primers bcfluG-5F and bcfluG-3R, and were transformed in B05.10, yielding 10 hygromycinresistant transformants. Two transformants that have undergone homologous integration (detected by diagnostic PCR as described above) were purified by two consecutive rounds of single-spore isolation. Homokaryotic mutants $(\Delta b c f l u G-\mathrm{T} 1$, $\Delta b c f l u G$-T8) were subjected to Southern blot analysis to rule out further ectopic integration events. For that, genomic DNA was digested with $S a c$ I and was blotted and hybridized with the 5 ' flank of bcfluG. As expected, single hybridizing fragments were obtained in all strains. The shift of the fragment sizes is due to the loss of SacI restriction sites in the replacement mutants. For generation of mutants overexpressing $b c f l u G$, the ORF was amplified with primers $b c f l u G$-PoliC-F and $b c f l u G-$ Tgluc-R and was assembled with the NcoI+NotI-digested plasmid pNDN-OGG (Schumacher 2012), yielding pNDNPoliC::bcfluG. The construct was transformed into B05.10 yielding eight nourseothricin-resistant transformants. Targeted integration at bcniaD was detected in transformants $\mathrm{OE}:$ : $b c f l u G-\mathrm{T} 2$, OE::bcfluG-T6, and OE::bcfluG-T8 by diagnostic PCR, using primers bcniaD-hi5F and Tgluc-hiF and bcniaDhi3R and natl-hiF (data not shown). Overexpression of $b c f l u G$ in the respective transformants was confirmed by Northern blot analyses (data not shown).

\section{Protein-protein interaction studies.}

Protein-protein interaction assays were performed according to the standard yeast two-hybrid method established by Fields and Song (1989). Proteins of interest were fused to Gal4activation and DNA-binding domains in vectors pAD-GAL42.1 and pBD-GAL4-Cam (Stratagene Corp.), respectively, and were expressed in Saccharomyces cerevisiae PJ69-4A (James et al. 1996). For vector cloning, genes of interest were amplified with primers containing overlaps with the Gal4-activation domain (AD-F, AD-R) or the Gal4-binding domain (BD-F, BD-R), using cDNA as template. Amplicons were cotransformed with SalI-digested plasmids pAD-GAL4-2.1 (selection for leucine prototrophy) or pBD-GAL4-Cam (selection for tryptophan prototrophy) in yeast strain PJ69-4A. Assembled vectors were isolated and transformed into Escherichia coli for amplification and sequencing. For interaction assays, Saccharomyces cerevisiae PJ69-4A was cotransformed with pAD-GAL4-2.1 and pBD-GAL4-Cam vectors according to a modified protocol of the lithium acetate method (Gietz et al. 1995). Selection took place on synthetic defined medium without leucine and tryptophan (SD-LW) for obtaining strains with both vectors. For drop tests, the strains were grown overnight in $5 \mathrm{ml}$ of liquid SD-LW and were adjusted to an optical density at $600 \mathrm{~nm}$ of 1 . Cells were starved in $1 \mathrm{M}$ sorbitol for $5 \mathrm{~h}$ at $30^{\circ} \mathrm{C}$ and dilutions of $1: 10$ were prepared. Ten-microliter droplets were spotted on solid $\mathrm{SD}-\mathrm{LW}+\mathrm{X}-\mathrm{Gal}$ and SD-LWH+3-AT (3-amino-1,2,4-triazole) media. Petri dishes were incubated for 3 days at $30^{\circ} \mathrm{C}$.

\section{Virulence assays.}

For infection assays on French bean (Phaseolus vulgaris cv. 90598), primary leaves of living plants were inoculated with $7.5-\mu 1$ droplets of conidial suspensions $\left(2 \times 10^{5}\right.$ conidia per milliliter of Gamborg B5 $+2 \%$ glucose) or undifferentiated mycelia (plugs from 3-day-old cultures on solid CM). Infected plants were incubated in plastic boxes at $22^{\circ} \mathrm{C}$ under natural illumination (daylight/night) conditions. Grape berries (Vitis vinifera) were inoculated with $10-\mu \mathrm{l}$ droplets of conidial suspensions $\left(5 \times 10^{5}\right.$ conidia per milliliter of potato dextrose broth). Infected berries were incubated in plastic boxes at $16^{\circ} \mathrm{C}$ under natural illumination conditions.

\section{Microarrays analyses.}

Conidial suspensions from wild-type B05.10 and mutant strains $\Delta$ bcvell and $\Delta b c l a e l$ were spread onto solid grape juice medium ( $\mathrm{pH}$ adjusted to $4 ; 3 \times 10^{6}$ conidia/plate) with cellophane overlays as described by Simon et al. (2013). The plates were incubated for $48 \mathrm{~h}$ at $23^{\circ} \mathrm{C}$ with an alternation of $10 \mathrm{~h}$ of white light (fluorescent tubes OSRAM L 36W/640) and $14 \mathrm{~h}$ of darkness in an environmental chamber (Sanyo MLR-350H). RNA from four biological replicates was extracted according to the total RNA isolation protocol described by Reid et al. (2006) but with $10 \mathrm{M}$ rather than $8 \mathrm{M} \mathrm{LiCl}$ and was resuspended in RNAse/DNAse-free water. The total RNA was then treated with the DNA-free kit (Ambion and Applied Biosystems) to remove any trace of DNA. Synthesis of double-stranded cDNA, Cy3labeling, and hybridization of microarrays were done at PartnerChip (Evry, France), using the procedures established by NimbleGen (Roche) and reagents from Invitrogen (Life technologies). To study the complete transcriptome of $B$. cinerea, NimbleGen 4-plex arrays with 62,478 60 mer-specific probes covering all the 20,885 predicted gene models and nonmapping expressed sequence tags (EST) of B. cinerea (Amselem et al. 2011) were used. The arrays also include 9,559 random probes as negative controls. Data processing, quality controls, and differential expression analysis were performed using ANAIS methods (Simon and Biot 2010). Genes whose intensity is over the threshold $(1.5 \times 95$ th percentile of random probes $)$ in at least one hybridization were considered expressed and were kept for further analyses. Genes with a corrected $P$ value $<0.05$ and more than a twofold change in transcript level (either in 
$\Delta$ bcvell or $\Delta$ bclael compared with the wild type) were considered as significantly DE. BlastN analyses for the 20,885 spotted genes (Amselem et al. 2011) (URGI database) were performed to identify the matching gene models derived from the resequencing approaches by Staats and van Kan (2012) (Broad Institute). By this, the number of DE genes was reduced, because incorrect gene models derived from discontinuous sequences were revised and, furthermore, previously nonmatching EST could be mapped. Cluster analyses were performed to highlight expression profiles for the DE genes. For this purpose, the $\log 2$-normalized intensities scaled by gene across the 12 hybridizations were clustered using Genesis tools (Sturn et al. 2002). Gene enrichment analyses were further performed with the GSEA toolkit (Mootha et al. 2003; Subramanian et al. 2005, 2007) to highlight significantly enriched functions in DE genes compared with the complete list of functionally annotated $B$. cinerea genes (Amselem et al. 2011). Details on the experiments, raw, and normalized values are available at NCBI Gene Expression Omnibus (accession number GSE63021). Furthermore, lists of DE genes are available in the URGI database.

\section{qRT-PCR analyses.}

Triplicate cultures of wild-type B05.10 and $\Delta b$ clae 1, OE:: bclae1, $\Delta$ bcvel1, and OE::bcvell mutants and the corresponding DNA-free RNA preparations were conducted as described for the microarray analysis. cDNA were obtained from 1,100 ng of total RNA by a retrotranscription step realized with M-MLV (Invitrogen) reverse transcription, according to the manufacturer's suggestions except that both oligo(dT) $(25 \mathrm{ng} / \mu \mathrm{l})$ and random primers $(25 \mathrm{ng} / \mu \mathrm{l})$ were used. The RT-qPCR reactions were performed on 1:10 diluted cDNA using MESA Green qPCR Mastermix Plus for SYBR Assay (Eurogentec) and the CFX96 Touch real-time PCR detection system (Bio-Rad). 'No Reverse Transcription' controls were realized once on cDNA samples and 'No Template Control' were made for each pair of primers in every run. The qRT-PCR program consisted of a preliminary 5 -min denaturation step at $95^{\circ} \mathrm{C}$, followed by 40 amplification cycles of $15 \mathrm{~s}$ of denaturation $\left(95^{\circ} \mathrm{C}\right)$ and $1 \mathrm{~min}$ of annealing and extension $\left(60^{\circ} \mathrm{C}\right)$. Analysis of the melting curves (increment of $0.1^{\circ} \mathrm{C} / \mathrm{min}$ ) of the final PCR products indicated the absence of contamination and primer dimers. The data were analyzed with CFX ManagerSoftware (Bio-Rad Laboratories, Inc.). Efficiency reactions of the pairs of primers were tested once on the cDNA sample and were all superior to $92 \%$. Gene expression levels were calculated following the $2^{-\Delta \Delta C T}$ method (Livak and Schmittgen 2001), using the real efficiency of the primers and the constitutively expressed actin gene (bcact 1 ) as a reference. Wild-type B05.10 was used as control.

\section{ACKNOWLEDGMENTS}

We thank B. Richter and J. Espino (WWU Münster) for their help in mutant screens, P. Le Pêcheur (INRA Grignon, France) for pathotests on grape berries, and S. Baulande and M. Duthieuw (PartnerChip, Evry, France) for the hybridization of the NimbleGen arrays.

\section{LITERATURE CITED}

Adams, T. H., Boylan, M. T., and Timberlake, W. E. 1988. brlA is necessary and sufficient to direct conidiophore development in Aspergillus nidulans. Cell 54:353-362.

Aghcheh, R. K., Druzhinina, I. S., and Kubicek, C. P. 2013. The putative protein methyltransferase LAE1 of Trichoderma atroviride is a key regulator of asexual development and mycoparasitism. PLoS ONE 8:e67144.

Ahmed, Y. L., Gerke, J., Park, H. S., Bayram, Ö., Neumann, P., Ni, M., Dickmanns, A., Kim, S. C., Yu, J. H., Braus, G. H., and Ficner, R. 2013. The velvet family of fungal regulators contains a DNA-binding domain

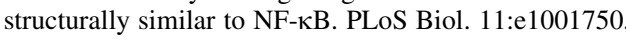

Amselem, J., Cuomo, C. A., van Kan, J. A., Viaud, M., Benito, E. P., Couloux, A., Coutinho, P. M., de Vries, R. P., Dyer, P. S., Fillinger, S., Fournier, E., Gout, L., Hahn, M., Kohn, L., Lapalu, N., Plummer, K. M., Pradier, J. M., Quévillon, E., Sharon, A., Simon, A., ten Have, A., Tudzynski, B., Tudzynski, P., Wincker, P., Andrew, M., Anthouard, V., Beever, R. E., Beffa, R., Benoit, I., Bouzid, O., Brault, B., Chen, Z., Choquer, M., Collémare, J., Cotton, P., Danchin, E. G., Da Silva, C. Gautier, A., Giraud, C., Giraud, T., Gonzalez, C., Grossetete, S., Güldener, U., Henrissat, B., Howlett, B. J., Kodira, C., Kretschmer, M., Lappartient, A., Leroch, M., Levis, C., Mauceli, E., Neuvéglise, C., Oeser, B., Pearson, M., Poulain, J., Poussereau, N., Quesneville, H., Rascle, C., Schumacher, J., Ségurens, B., Sexton, A., Silva, E., Sirven, C., Soanes, D. M., Talbot, N. J., Templeton, M., Yandava, C., Yarden, O., Zeng, Q., Rollins, J. A., Lebrun, M. H., and Dickman, M. 2011. Genomic analysis of the necrotrophic fungal pathogens Sclerotinia sclerotiorum and Botrytis cinerea. PLoS Genet. 7:e1002230.

Bailey, L. A., and Ebbole, D. J. 1998. The fluffy gene of Neurospora crassa encodes a Gal4p-type C6 zinc cluster protein required for conidial development. Genetics 148:1813-1820.

Bayram, O., and Braus, G. H. 2012. Coordination of secondary metabolism and development in fungi: The velvet family of regulatory proteins FEMS (Fed. Eur. Microbiol. Soc.) Microbiol. Rev. 36:1-24.

Bayram, O., Krappmann, S., Ni, M., Bok, J. W., Helmstaedt, K., Valerius, O., Braus-Stromeyer, S., Kwon, N. J., Keller, N. P., Yu, J. H., and Braus, G. H. 2008. VelB/VeA/LaeA complex coordinates light signal with fungal development and secondary metabolism. Science 320:1504-1506.

Bok, J. W., and Keller, N. P. 2004. LaeA, a regulator of secondary metabolism in Aspergillus spp. Eukaryot. Cell 3:527-535.

Bok, J. W., Balajee, S. A., Marr, K. A., Andes, D., Nielsen, K. F., Frisvad, J. C., and Keller, N. P. 2005. LaeA, a regulator of morphogenetic fungal virulence factors. Eukaryot. Cell 4:1574-1582.

Brakhage, A. A. 2013. Regulation of fungal secondary metabolism. Nat. Rev. Microbiol. 11:21-32.

Campbell, M. A., Rokas, A., and Slot, J. C. 2012. Horizontal transfer and death of a fungal secondary metabolic gene cluster. Genome Biol. Evol. 4:289-293.

Canessa, P., Schumacher, J., Hevia, M. A., Tudzynski, P., and Larrondo, L. F. 2013. Assessing the effects of light on differentiation and virulence of the plant pathogen Botrytis cinerea: Characterization of the white collar complex. PLoS ONE 8:e84223.

Cenis, J. L. 1992. Rapid extraction of fungal DNA for PCR amplification. Nucleic Acids Res. 20:2380.

Chang, P. K., Scharfenstein, L. L., Mack, B., and Ehrlich, K. C. 2012. Deletion of the Aspergillus flavus orthologue of $A$. nidulans fluG reduces conidiation and promotes production of sclerotia but does not abolish aflatoxin biosynthesis. Appl. Environ. Microbiol. 78:7557-7563.

Chang, P. K., Scharfenstein, L. L., Li, P., and Ehrlich, K. C. 2013. Aspergillus flavus VelB acts distinctly from VeA in conidiation and may coordinate with FluG to modulate sclerotial production. Fungal Genet. Biol. 58-59:71-79.

Choquer, M., Fournier, E., Kunz, C., Levis, C., Pradier, J. M., Simon, A., and Viaud, M. 2007. Botrytis cinerea virulence factors: New insights into a necrotrophic and polyphageous pathogen. FEMS (Fed. Eur. Microbiol Soc.) Microbiol. Lett. 277:1-10.

Collado, I. G., Sánchez, A. J., and Hanson, J. R. 2007. Fungal terpene metabolites: Biosynthetic relationships and the control of the phytopathogenic fungus Botrytis cinerea. Nat. Prod. Rep. 24:674-686.

Colot, H. V., Park, G., Turner, G. E., Ringelberg, C., Crew, C. M., Litvinkova, L., Weiss, R. L., Borkovich, K. A., and Dunlap, J. C. 2006. A high-throughput gene knockout procedure for Neurospora reveals functions for multiple transcription factors. Proc. Natl. Acad. Sci. USA 103:10352-10357.

D'Souza, C. A., Lee, B. N., and Adams, T. H. 2001. Characterization of the role of the FluG protein in asexual development of Aspergillus nidulans. Genetics 158:1027-1036.

Dalmais, B., Schumacher, J., Moraga, J., LE Pêcheur, P., Tudzynski, B., Collado, I. G., and Viaud, M. 2011. The Botrytis cinerea phytotoxin botcinic acid requires two polyketide synthases for production and has a redundant role in virulence with botrydial. Mol. Plant Pathol. 12:564-579.

Doss, R. P., Deisenhofer, J., Krug von Nidda, H. A., Soeldner, A. H., and McGuire, R. P. 2003. Melanin in the extracellular matrix of germlings of Botrytis cinerea. Phytochemistry 63:687-691.

Epton, H. A. S., and Richmond, D. V. 1980. Formation, structures and germination of conidia. Pages 41-83 in The Biology of Botrytis cinerea. J. R. Coley-Smith, K. Verhoeff, and W. R. Jarvis, eds. Academic Press, London.

Espino, J. J., Gutiérrez-Sánchez, G., Brito, N., Shah, P., Orlando, R., and González, C. 2010. The Botrytis cinerea early secretome. Proteomics 10: 3020-3034 
Faretra, F., and Antonacci, E. 1987. Production of apothecia of Botryotinia fuckeliana (de Bary) Whetz under controlled environmental conditions. Phytopathol. Mediterr. 26:29-35.

Faretra, F., Antonacci, E., and Pollastro, S. 1988. Sexual behaviour and mating system of Botryotinia fuckeliana, teleomorph of Botrytis cinerea. J. Gen. Microbiol. 134:2543-2550.

Fields, S., and Song, O. 1989. A novel genetic system to detect proteinprotein interactions. Nature 340:245-246.

Gao, Q., and Garcia-Pichel, F. 2011. Microbial ultraviolet sunscreens. Nat. Rev. Microbiol. 9:791-802.

Gietz, R. D., Schiestl, R. H., Willems, A. R., and Woods, R. A. 1995. Studies on the transformation of intact yeast cells by the LiAc/SS-DNA/ PEG procedure. Yeast 11:355-360.

Godoy, G., Steadman, J. R., Dickman, M. B., and Dam, R. 1990. Use of mutants to demonstrate the role of oxalicacid in pathogenicity of Sclerotinia sclerotiorum on Phaseolus vulgaris. Physiol. Mol. Plant Pathol. 37:179-191.

Han, Y., Joosten, H. J., Niu, W., Zhao, Z., Mariano, P. S., McCalman, M., van Kan, J., Schaap, P. J., and Dunaway-Mariano, D. 2007. Oxaloacetate hydrolase, the $\mathrm{C}-\mathrm{C}$ bond lyase of oxalate secreting fungi. J. Biol. Chem. 282:9581-9590

Hoff, B., Kamerewerd, J., Sigl, C., Mitterbauer, R., Zadra, I., Kürnsteiner, H., and Kück, U. 2010. Two components of a velvet-like complex control hyphal morphogenesis, conidiophore development, and penicillin biosynthesis in Penicillium chrysogenum. Eukaryot. Cell 9:1236-1250.

Hoffmeister, D., and Keller, N. P. 2007. Natural products of filamentous fungi: Enzymes, genes, and their regulation. Nat. Prod. Rep. 24:393-416.

James, P., Halladay, J., and Craig, E. A. 1996. Genomic libraries and a host strain designed for highly efficient two-hybrid selection in yeast. Genetics 144:1425-1436.

Kim, H. J., Han, J. H., Kim, K. S., and Lee, Y. H. 2014. Comparative functional analysis of the velvet gene family reveals unique roles in fungal development and pathogenicity in Magnaporthe oryzae. Fungal Genet. Biol. 66:33-43.

Kroken, S., Glass, N. L., Taylor, J. W., Yoder, O. C., and Turgeon, B. G. 2003. Phylogenomic analysis of type I polyketide synthase genes in pathogenic and saprobic ascomycetes. Proc. Natl. Acad. Sci. USA 100: $15670-15675$

Lee, B. N., and Adams, T. H. 1994. The Aspergillus nidulans fluG gene is required for production of an extracellular developmental signal and is related to prokaryotic glutamine synthetase I. Genes Dev. 8:641-651.

Liang, X., Liberti, D., Li, M., Kim, Y. T., Hutchens, A., Wilson, R., and Rollins, J. A. 2014. Oxaloacetate acetylhydrolase gene mutants of Sclerotinia sclerotiorum do not accumulate oxalic acid, but do produce limited lesions on host plants. Mol. Plant Pathol. 10.1111/mpp.12211. Published online.

Livak, K. J., and Schmittgen, T. D. 2001. Analysis of relative gene expression data using real-time quantitative PCR and the 2(-Delta Delta C(T)) Method. Methods 25:402-408

Massaroli, M., Moraga, J., Bastos Borges, K., Ramírez-Fernández, J., Viaud, M., González Collado, I., Durán-Patrón, R., and HernándezGalán, R. 2013. A shared biosynthetic pathway for botcinins and botrylactones revealed through gene deletions. ChemBioChem 14: 132-136.

Mootha, V. K., Lindgren, C. M., Eriksson, K. F., Subramanian, A., Sihag, S., Lehar, J., Puigserver, P., Carlsson, E., Ridderstråle, M., Laurila, E., Houstis, N., Daly, M. J., Patterson, N., Mesirov, J. P., Golub, T. R. Tamayo, P., Spiegelman, B., Lander, E. S., Hirschhorn, J. N., Altshuler, D., and Groop, L. C. 2003. PGC-1alpha-responsive genes involved in oxidative phosphorylation are coordinately downregulated in human diabetes. Nat. Genet. 34:267-273.

Olmedo, M., Ruger-Herreros, C., and Corrochano, L. M. 2010. Regulation by blue light of the fluffy gene encoding a major regulator of conidiation in Neurospora crassa. Genetics 184:651-658.

Oritani, T., and Kiyota, H. 2003. Biosynthesis and metabolism of abscisic acid and related compounds. Nat. Prod. Rep. 20:414-425.

Palmer, J. M., Theisen, J. M., Duran, R. M., Grayburn, W. S., Calvo, A. M., and Keller, N. P. 2013. Secondary metabolism and development is mediated by LlmF control of VeA subcellular localization in Aspergillus nidulans. PLoS Genet. 9:e1003193.

Park, H. S., Nam, T. Y., Han, K. H., Kim, S. C., and Yu, J. H. 2014. VelC positively controls sexual development in Aspergillus nidulans. PLoS ONE 9:e89883.

Paul, W. R. C. 1929. A comparative morphological and physiological study of a number of strains of Botrytis cinerea Pers. with special reference to their virulence. Trans. Br. Mycol. Soc. 14:118-134.

Pinedo, C., Wang, C. M., Pradier, J. M., Dalmais, B., Choquer, M., Le Pêcheur, P., Morgant, G., Collado, I. G., Cane, D. E., and Viaud, M.
2008. Sesquiterpene synthase from the botrydial biosynthetic gene cluster of the phytopathogen Botrytis cinerea. ACS Chem. Biol. 3: 791-801.

Pontecorvo, G., Roper, J. A., Hemmons, L. M., MacDonald, K. D., and Bufton, A. W. J. 1953. The genetics of Aspergillus nidulans. Adv. Genet 5:141-238.

Purschwitz, J., Müller, S., Kastner, C., Schöser, M., Haas, H., Espeso, E. A., Atoui, A., Calvo, A. M., and Fischer, R. 2008. Functional and physical interaction of blue- and red-light sensors in Aspergillus nidulans. Curr. Biol. 18:255-259.

Quidde, T., Osbourn, A. E., and Tudzynski, P. 1998. Detoxification of $\alpha$-tomatine by Botrytis cinerea. Physiol. Mol. Plant Pathol. 52:151-165.

Reid, K. E., Olsson, N., Schlosser, J., Peng, F., and Lund, S. T. 2006. An optimized grapevine RNA isolation procedure and statistical determination of reference genes for real-time RT-PCR during berry development. BMC Plant Biol. 6:27.

Reino, J. L., Hernandez-Galan, R., Duran-Patron, R., and Collado, I. G. 2004. Virulence-toxin production relationship in isolates of the plant pathogenic fungus Botrytis cinerea. J. Phytopathol. 152:563-566.

Rossi, F. R., Gárriz, A., Marina, M., Romero, F. M., Gonzalez, M. E., Collado, I. G., and Pieckenstain, F. L. 2011. The sesquiterpene botrydial produced by Botrytis cinerea induces the hypersensitive response on plant tissues and its action is modulated by salicylic acid and jasmonic acid signaling. Mol. Plant Microbe Interact. 24:888-896.

Ruger-Herreros, C., Rodríguez-Romero, J., Fernández-Barranco, R., Olmedo, M., Fischer, R., Corrochano, L. M., and Canovas, D. 2011. Regulation of conidiation by light in Aspergillus nidulans. Genetics 188:809-822.

Saikia, S., Nicholson, M. J., Young, C., Parker, E. J., and Scott, B. 2008 The genetic basis for indole-diterpene chemical diversity in filamentous fungi. Mycol. Res. 112, Pt 2:184-199.

Sambrook, J., Fritsch, E. F., and Maniatis, T. 1989. Molecular Cloning: a Laboratory Manual, 2nd Ed. Cold Spring Harbor Laboratory Press, Cold Spring Harbor, NY, U.S.A..

Schumacher, J. 2012. Tools for Botrytis cinerea: New expression vectors make the gray mold fungus more accessible to cell biology approaches. Fungal Genet. Biol. 49:483-497.

Schumacher, J., Pradier, J. M., Simon, A., Traeger, S., Moraga, J., Collado, I. G., Viaud, M., and Tudzynski, B. 2012. Natural variation in the VELVET gene bcvel1 affects virulence and light-dependent differentiation in Botrytis cinerea. PLoS ONE 7:e47840.

Schumacher, J., Gautier, A., Morgant, G., Studt, L., Ducrot, P. H., Le Pêcheur, P., Azeddine, S., Fillinger, S., Leroux, P., Tudzynski, B., and Viaud, M. 2013. A functional bikaverin biosynthesis gene cluster in rare strains of Botrytis cinerea is positively controlled by VELVET. PLoS ONE 8:e53729.

Schumacher, J., Simon, A., Cohrs, K. C., Viaud, M., and Tudzynski, P. 2014. The transcription factor BcLTF1 regulates virulence and light responses in the necrotrophic plant pathogen Botrytis cinerea. PLoS Genet. 10:e1004040

Siewers, V., Smedsgaard, J., and Tudzynski, P. 2004. The P450 monooxygenase BcABA1 is essential for abscisic acid biosynthesis in Botrytis cinerea. Appl. Environ. Microbiol. 70:3868-3876.

Siewers, V., Viaud, M., Jimenez-Teja, D., Collado, I. G., Gronover, C. S., Pradier, J. M., Tudzynski, B., and Tudzynski, P. 2005. Functional analysis of the cytochrome P450 monooxygenase gene bcbot1 of Botrytis cinerea indicates that botrydial is a strain-specific virulence factor. Mol. Plant Microbe Interact. 18:602-612.

Siewers, V., Kokkelink, L., Smedsgaard, J., and Tudzynski, P. 2006. Identification of an abscisic acid gene cluster in the grey mold Botrytis cinerea. Appl. Environ. Microbiol. 72:4619-4626.

Simon, A., and Biot, E. 2010. ANAIS: Analysis of NimbleGen arrays interface. Bioinformatics 26:2468-2469.

Simon, A., Dalmais, B., Morgant, G., and Viaud, M. 2013. Screening of a Botrytis cinerea one-hybrid library reveals a $\mathrm{Cys}_{2} \mathrm{His}_{2}$ transcription factor involved in the regulation of secondary metabolism gene clusters. Fungal Genet. Biol. 52:9-19.

Staats, M., and van Kan, J. A. 2012. Genome update of Botrytis cinerea strains B05.10 and T4. Eukaryot. Cell 11:1413-1414.

Stefanato, F. L., Abou-Mansour, E., van Kan, J., Metraux, J. P., and Schoonbeek, H. J. 2008. Oxaloacetate acetylhydrolase is responsible for oxalic acid production in Botrytis cinerea and required for lesion expansion on some, but not on most host plants. Page 24 in: 3rd Botrytis Genome Workshop, Third Session, Pathogenicity, Tenerife, Spain. http:// botrytis.webs.ull.es/BookAbst.pdf. Published online.

Stewart, T. M., and Long, P. G. 1987. Sporulation of Botrytis cinerea in the dark. N. Zeal. J. Exp. Agricul. 15:389-392.

Stinnett, S. M., Espeso, E. A., Cobeño, L., Araújo-Bazán, L., and Calvo, A. M. 2007. Aspergillus nidulans VeA subcellular localization is 
dependent on the importin alpha carrier and on light. Mol. Microbiol. 63: 242-255.

Sturn, A., Quackenbush, J., and Trajanoski, Z. 2002. Genesis: Cluster analysis of microarray data. Bioinformatics 18:207-208.

Subramanian, A., Tamayo, P., Mootha, V. K., Mukherjee, S., Ebert, B. L., Gillette, M. A., Paulovich, A., Pomeroy, S. L., Golub, T. R., Lander, E. S., and Mesirov, J. P. 2005. Gene set enrichment analysis: a knowledge-based approach for interpreting genome-wide expression profiles. Proc. Natl. Acad. Sci. USA 102:15545-15550.

Subramanian, A., Kuehn, H., Gould, J., Tamayo, P., and Mesirov, J. P. 2007. GSEA-P: A desktop application for gene set enrichment analysis. Bioinformatics 23:3251-3253.

Tani, H., Koshino, H., Sakuno, E., and Nakajima, H. 2005. Botcinins A, B, $\mathrm{C}$, and D, metabolites produced by Botrytis cinerea, and their antifungal activity against Magnaporthe grisea, a pathogen of rice blast disease. J. Nat. Prod. 68:1768-1772.

Tani, H., Koshino, H., Sakuno, E., Cutler, H. G., and Nakajima, H. 2006. Botcinins $\mathrm{E}$ and $\mathrm{F}$ and Botcinolide from Botrytis cinerea and structural revision of botcinolides. J. Nat. Prod. 69:722-725.

van Kan, J. A. 2006. Licensed to kill: the lifestyle of a necrotrophic plant pathogen. Trends Plant Sci. 11:247-253.

Wiemann, P., and Keller, N. P. 2014. Strategies for mining fungal natural products. J. Ind. Microbiol. Biotechnol. 41:301-313.

Wiemann, P., Willmann, A., Straeten, M., Kleigrewe, K., Beyer, M., Humpf, H. U., and Tudzynski, B. 2009. Biosynthesis of the red pigment bikaverin in Fusarium fujikuroi: genes, their function and regulation. Mol. Microbiol. 72:931-946.

Wiemann, P., Brown, D. W., Kleigrewe, K., Bok, J. W., Keller, N. P., Humpf, H. U., and Tudzynski, B. 2010. FfVel1 and FfLae1, components of a velvet-like complex in Fusarium fujikuroi, affect differentiation, secondary metabolism and virulence. Mol. Microbiol. 77:972-994.

Williamson, B., Tudzynski, B., Tudzynski, P., and van Kan, J. A. 2007. Botrytis cinerea: The cause of grey mould disease. Mol. Plant Pathol. 8 561-580.

Wu, D., Oide, S., Zhang, N., Choi, M. Y., and Turgeon, B. G. 2012. ChLae1 and ChVel1 regulate T-toxin production, virulence, oxidative stress response, and development of the maize pathogen Cochliobolus heterostrophus. PLoS Pathog. 8:e1002542.

Yager, L. N., Lee, H. O., Nagle, D. L., and Zimmerman, J. E. 1998. Analysis of fluG mutations that affect light-dependent conidiation in Aspergillus nidulans. Genetics 149:1777-1786.

Yang, Q., Chen, Y., and Ma, Z. 2013. Involvement of BcVeA and BcVelB in regulating conidiation, pigmentation and virulence in Botrytis cinerea. Fungal Genet. Biol. 50:63-71.

Zeun, R., and Buchenauer, H. 1985. Effect of tricyclazole on production and melanin contents of sclerotia of Botrytis cinerea. Phytopathol. Z. 112:259-267.

\section{AUTHOR-RECOMMENDED INTERNET RESOURCES}

Broad Institute Botrytis cinerea database: www.broadinstitute.org/annotation/genome/botrytis_cinerea GSEA toolkit: www.broadinstitute.org/gsea

NCBI Gene Expression Omnibus database: www.ncbi.nlm.nih.gov/geo URGI Plant and Data Integration database: urgi.versailles.inra.fr/Data/Transcriptome 